\title{
Protoporphyrin IX sensitized titanium oxide gel electrode
}

\author{
Shuxin Tan, Bin Su, Christophe Roussel, Hubert H. Girault * \\ Laboratoire d'Electrochimie Physique et Analytique, Ecole Polytechnique Fédérale de Lausanne, Station 6, CH-1015 Lausanne, Switzerland
}

Received 2 March 2007; received in revised form 19 June 2007; accepted 23 June 2007

Available online 3 July 2007

Dedicated to Professor Michael Grätzel

\begin{abstract}
Titanium oxide $(\mathrm{Ti}(\mathrm{O}))$ xerogel films functionalized by protoporphyrin IX (PPIX) and ferrocene carboxylic acid (FCA) were deposited on indium tin oxide (ITO) electrodes following a sol-gel synthesis. PPIX and FCA were first complexed to titanium oxide precursors, which were then subjected to hydrolysis to obtain a homogenous $\mathrm{Ti}(\mathrm{O})$ polymeric network gel doped with PPIX and FCA. The Ti(O) film cast on the ITO electrode has been characterized by UV-Vis absorption, X-ray photoelectron spectroscopy (XPS), scanning electron microscopy and cyclic voltammetry. Illumination of the PPIX doped Ti(O) films on the ITO electrode immersed in aqueous electrolytes onsets photoinduced electron transfer reactions, and a cathodic photocurrent was observed in most cases. This photocurrent response was investigated in detail using a kinetic model. Preliminary investigations of oxygen reduction, lithium and proton insertion into the $\mathrm{Ti}(\mathrm{O})$ film have also been carried out.
\end{abstract}

(c) 2007 Elsevier B.V. All rights reserved.

Keywords: Titanium oxide gel; PPIX; FCA; Photocurrent; Electron transfer

\section{Introduction}

Converting sunlight into chemical or electrical energy remains one of the major social issues. So far, various solar-to-energy conversion systems have been proposed with different conversion efficiencies. For example, silicon-based inorganic solar cells featuring high conversion efficiencies have been produced. However, the energy cost of high-purity silicon still hinders the mass production of silicon solar cells. In 1990s', substantial advances in the conversion efficiency have also been achieved in dye-sensitized solar cells $[1,2]$, also called Grätzel's cells, by using mesoporous titanium dioxide films, which feature a large surface area available for the dye loading and therefore improve the light harvesting.

In nature, the photosynthetic process converts solar energy into chemical energy, the mechanism by which liv-

\footnotetext{
* Corresponding author. Tel.: +41 21693 3151; fax: +41 216933667

E-mail address: hubert.girault@epfl.ch (H.H. Girault).
}

ing organisms on the earth derive their energy. Therefore, an alternative way to realize solar-to-energy conversion is to mimic natural photosynthesis, in which the sequential event of photoinduced energy and electron transfer between an electron donor and an electron acceptor occurs at the biomembrane. Based on this natural principle, various types of molecular donor-acceptor assembly systems have been designed towards highly efficient photoelectric conversion [3-17]. Indeed, linked donor-acceptor compounds can produce a long-lived charge separated state with a high quantum yield, through which an efficient conversion of light to chemical and/or electrical energy can be achieved. Such typical compounds include diads of covalently linked porphyrin-fullerene molecules. Their photoelectrochemical properties in the molecular assemblies fabricated on the electrode surface have been extensively studied in recent years $[8,13,14,18-22]$. This system has been proposed for the development of efficient molecular photovoltaic devices. However, the chemical synthesis of these organic compounds is sophisticated and time-consuming. Alternatively, another method to incorporate 
donor-acceptor compounds into molecular assemblies toward light energy conversion has been developed using surface sol-gel processes [23-25]. Typically, an indium tin oxide (ITO) coated glass is first derivatized by an electron acceptor (for example, fullerene and viologen) through self-assembling, then a titanium oxide $(\mathrm{Ti}(\mathrm{O}))$ layer is generated by hydrolysis of titanium(IV) $n$-butoxide, and finally a sensitizer (for example, porphyrins) layer is deposited. This method does not require any sophisticated preparation procedures, and a variety of electron donor-acceptor pairs can be incorporated in the sol-gel structure.

In the present work, titanium oxide xerogel films functionalized by protoporphyrin IX (PPIX) and ferrocene carboxylic acid (FCA) were prepared on ITO electrodes using an organic phase sol-gel process with acetylacetone as the complexing ligand and inhibitor. The $\mathrm{Ti}(\mathrm{O})$ films on ITO electrodes were characterized by UV-Vis absorption, $\mathrm{X}$ ray photoelectron spectroscopy, scanning electron microscopy and cyclic voltammetry. The photoelectrochemical responses of PPIX sensitized titanium oxide films have been investigated in aqueous electrolytes. A kinetic model was also proposed to analyze the photocurrent responses. The rate constants of electron transfer between the PPIX species and the ITO electrode, as well as that of the quenching of PPIX excited state by FCA inside the film, were found to depend on the applied potential. Preliminary investigation into the oxygen reduction, ionic insertion into the $\mathrm{Ti}(\mathrm{O})$ network has also been carried out.

\section{Experimental}

\subsection{Chemicals}

All chemicals were used as received without further purification. Acetylacetone (AA, $\geqslant 99.5 \%$ ), ferrocene carboxylic acid (FCA, $\geqslant 97 \%$ ), 1-propanol ( $\mathrm{PrOH},>99 \%)$, ethanol (EtOH, $\sim 96 \%$ ), sodium sulfate $\left(\mathrm{Na}_{2} \mathrm{SO}_{4},>99 \%\right)$ and lithium chloride $(\mathrm{LiCl},>99 \%)$ were bought from Fluka. Acetone $(\geqslant 99.5 \%)$ and sodium hydroxide $(\mathrm{NaOH}, \geqslant 99 \%)$ were obtained from Riedel de Haën and Merck, respectively. Titanium(IV) $n$-butoxide ( $\left.\mathrm{Ti}(\mathrm{OBu})_{4}, 99 \%\right)$ and Protoporphyrin IX (PPIX, >97\%) were purchased from Acros and Frontier Scientific Inc., respectively. Ultrapure water $(18.2 \mathrm{M} \Omega \mathrm{cm})$ was obtained from a Milli-Q system. Indium tin oxide coated glass (resistance $<20 \Omega$ /square) was obtained from Merck. Fig. 1 illustrates the molecular structures of $\mathrm{Ti}(\mathrm{OBu})_{4}$, FCA and PPIX.

\subsection{Preparation of the titanium oxide film coated ITO electrodes}

The titanium oxide $(\mathrm{Ti}(\mathrm{O}))$ sol-gel material was prepared by a sol-gel process, basically in two steps: complexation and hydrolysis-condensation. Accordingly, two parameters: the complexation ratio $(x=$ complexing ligand $/ \mathrm{Ti}, \mathrm{mol} / \mathrm{mol})$ and the hydrolysis ratio $\left(H=\mathrm{H}_{2} \mathrm{O} /\right.$ $\mathrm{Ti}, \mathrm{mol} / \mathrm{mol}$ ) were adjusted to obtained suitable hydride

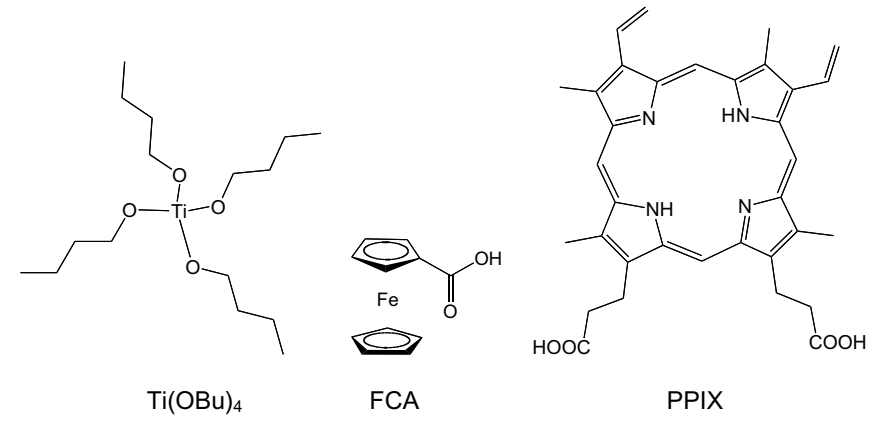

Fig. 1. Molecular structures of $\mathrm{Ti}(\mathrm{OBu})_{4}, \mathrm{FCA}$ and PPIX.

materials. As reported previously, these two parameters determine the final products obtained, molecular clusters, sols, gels or precipitates [26]. Typically, the first step consists of the complexation of $\mathrm{Ti}(\mathrm{OBu})_{4}$ by either FCA at $10 \%$, PPIX at $1 \%$ or PPIX and FCA at $1 \%$ and $10 \%$, which was followed by AA complexation at $40 \%$ (AA/Ti, mol/ mol). The complexation step was performed in solvent free conditions, thanks to the liquid nature of $\mathrm{Ti}(\mathrm{OBu})_{4}$ [27]. Both FCA/PPIX and AA are expected to complex with titanium atom through nucleophilic substitution, which increases the coordination number of titanium and correspondingly decreases its chemical reactivity towards hydrolysis-condensation. It is well known that metal alkoxide precursors are highly reactive towards hydrolysis and the stabilization by complexation is necessary in order to avoid the fast condensation to form precipitates [28,29]. In fact, AA here also functions as the inhibitor since the alkoxy groups are always hydrolyzed prior to the complexing ligand [28]. The resulting mixture after the complexing step was first diluted by $\mathrm{PrOH}$ in a volume ratio 1:10 (Ti/PrOH) and then hydrolyzed by dropwise addition of water with a hydrolysis ratio $(H)$ of 9.5 . The detailed synthetic procedures to prepare different sols are given in the supporting information (S.1).

The indium tin oxide (ITO) coated glasses were cleaned by sonication in acetone, ethanol and saturated $\mathrm{NaOH}$ ethanol solution, respectively, then rinsed thoroughly by ethanol and water and dried under an argon stream. The freshly prepared titanium oxide sols were deposited on the ITO electrode by doctor blading, followed by solvent evaporation in about 30 seconds to form a thin film with a thickness of $\sim 200 \mathrm{~nm}$ measured by profilometry. For simplicity, the $\mathrm{Ti}(\mathrm{O})$ film coated ITO electrodes in the presence of different doping compounds are denoted as ITO/ $\mathrm{Ti}(\mathrm{O})$, ITO/Ti(O)/FCA, ITO/Ti(O)/PPIX and ITO/ $\mathrm{Ti}(\mathrm{O}) /$ PPIX-FCA, respectively.

\subsection{Instrumentation}

Optical characterization of the $\mathrm{Ti}(\mathrm{O})$ film coated ITO electrodes was performed on a Laborlux D microscope from Leitz with a magnification of 50. The UV-Vis absorption spectroscopic measurements were recorded on an Ocean Optics CHEM2000-UV-Vis spectrometer. Microscopic 


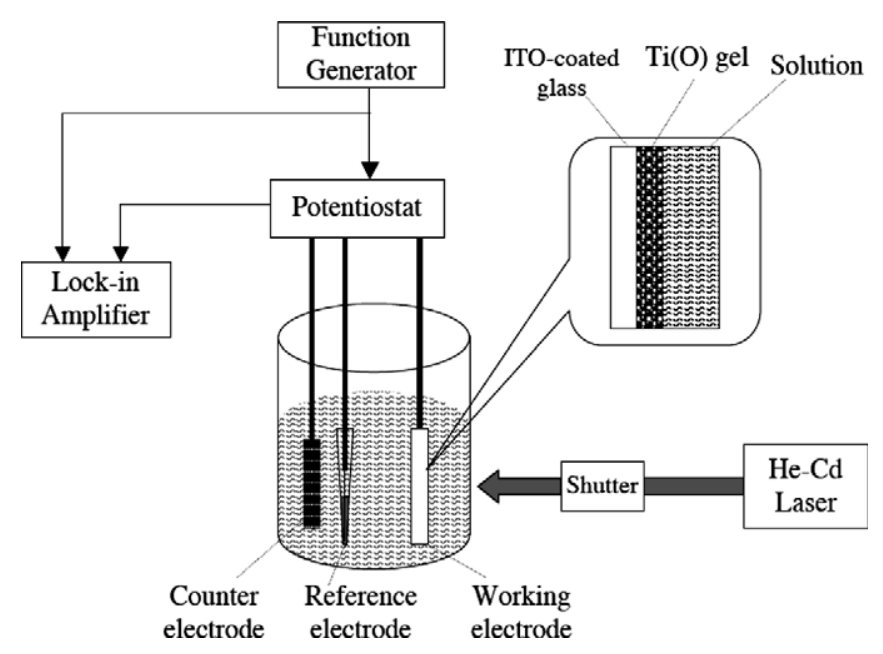

Fig. 2. Schematic illustration of the experimental apparatus for photocurrent transient measurements.

micrographs of the $\mathrm{Ti}(\mathrm{O})$ coated ITO electrodes were obtained from a FEI XL30 Sirion scanning electron microscope (SEM). The electrodes were dried under low pressure $\left(10^{-6}-10^{-7} \mathrm{mbar}\right)$ and a layer of amorphous carbon $(\sim 20 \mathrm{~nm})$ was deposited prior to SEM observations. The different films thicknesses were assessed by profilometry carried out on an Alpha Step 200 from Tencor instrument. $5 \mathrm{~mm}$ of the modified electrodes was scanned at $0.2 \mathrm{~s} \mathrm{~m}^{-1}$ with an instrumental precision of $\pm 5 \mathrm{~nm}$. X-ray photoelectron spectroscopy (XPS) was performed on a Kratos Axis Ultra apparatus (monochromatic $\mathrm{Al} \mathrm{K} \alpha, 15 \mathrm{kV}, 150 \mathrm{~W}$ ).

The cyclic voltammetric measurements were carried out on an Autolab PGSTAT 12 workstation (Metrohm) in water containing $0.1 \mathrm{M} \mathrm{LiCl}$, using a platinum counter electrode and an $\mathrm{Ag} / \mathrm{AgCl}$ (sat. $\mathrm{KCl}$ ) reference electrode. Photocurrent measurements were performed on a homemade system under potentiostatic conditions, with the external potential being supplied by a Hi-Tek waveform generator (Hi-Tek Instruments PP-R1). The $\mathrm{Ti}(\mathrm{O})$ modified ITO electrode was illuminated with a beam line of $442 \mathrm{~nm}$ from a He-Cd laser (Omnichrome S74) with a photon flux of $2.5 \times 10^{16} \mathrm{~cm}^{-2} \mathrm{~s}^{-1}$ determined by a calibrated power meter (Gentec, TPM-310). An optical shutter (Newport) was used to control the illumination time. The current was recorded with a SR830 lock-in amplifier (Stanford Research Systems). The potentiostatic control and the data acquisition were accomplished by means of custom Labview programs. The electrochemical and photoelectrochemical apparatus has been schematically illustrated in Fig. 2.

\section{Results and discussion}

\subsection{Characterization of the titanium oxide gels}

The titanium oxide gel coated on the ITO electrode was first characterized by UV-Vis absorption. Shown in Fig. 3a are the absorption spectra of the ITO/Ti(O) and ITO/
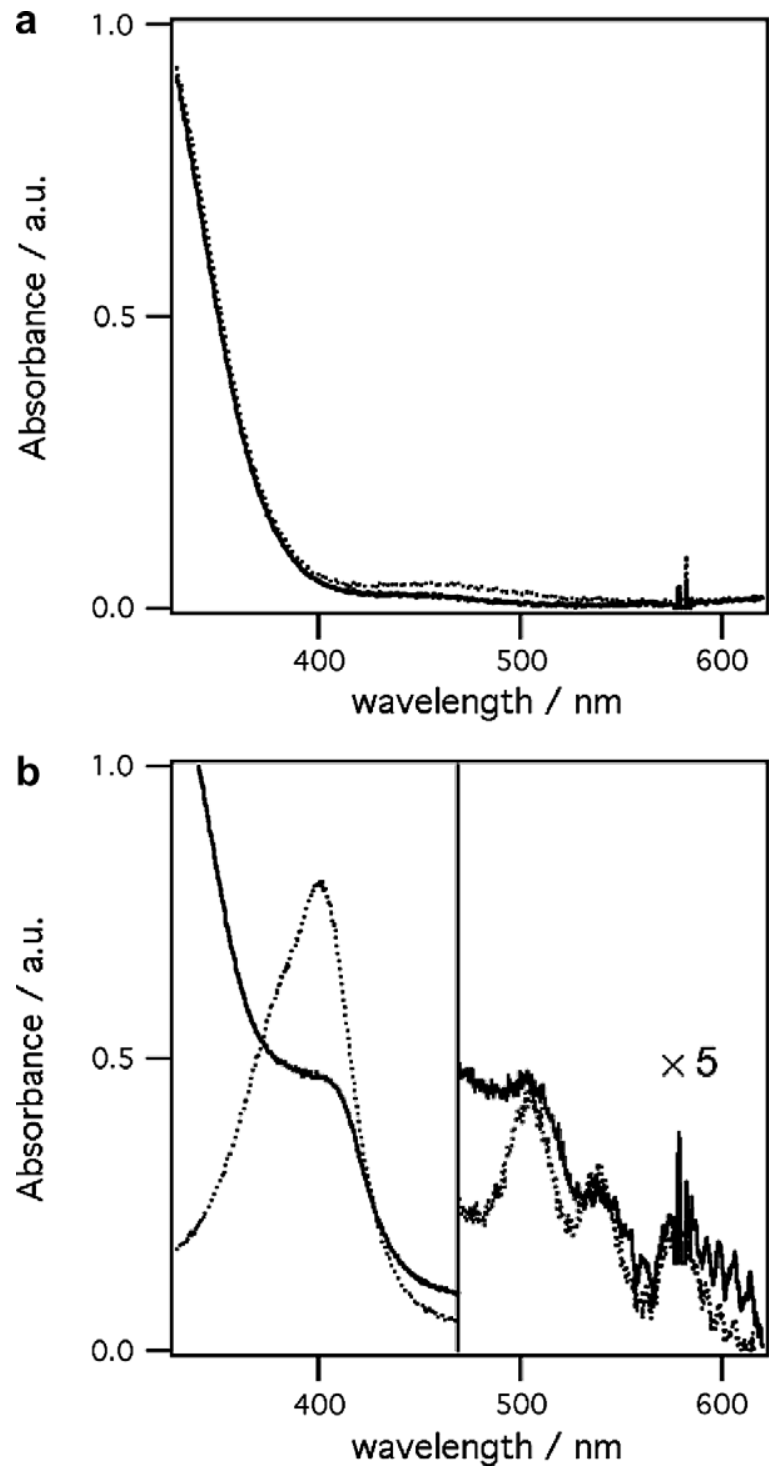

Fig. 3. (a) UV-Vis absorption spectra of the ITO/Ti(O) electrode (solid line) and the ITO/Ti(O)/FCA electrode (dotted line). (b) UV-Vis absorption spectra of the ITO/Ti(O)/PPIX-FCA electrode (solid line) and PPIX dilute solution (dotted line).

$\mathrm{Ti}(\mathrm{O}) / \mathrm{FCA}$ electrodes. The absorption onset of the ITO/ $\mathrm{Ti}(\mathrm{O})$ is around $400 \mathrm{~nm}$, which is close to the band-gap energy of the solid phase $\mathrm{TiO}_{2}$ of $3.0-3.2 \mathrm{eV}$. This indicates that although the gel is fundamentally different from $\mathrm{TiO}_{2}$ solids, certain similarities of their properties may exist. The light absorption results in the electron transition from the valence band of the $\mathrm{O}^{2-} 2 \mathrm{p}$ orbital to the conduction band of the $\mathrm{Ti}^{4+} 3 \mathrm{~d}$ orbital [30]. It can also be observed from Fig. 3a that the absorption spectrum of the ITO/Ti(O)/ FCA electrode is well overlapped with that of the ITO/ $\mathrm{Ti}(\mathrm{O})$ electrode, which means that the introduction of FCA into the gel does not change the optical characteristic of the gel. In contrast, when PPIX is incorporated, some new absorption bands that are relative to PPIX appear, as displayed in Fig. 3b. The intense absorption shoulder 
that lies at $\sim 400 \mathrm{~nm}$ is the Soret band, which is the origin of the second singlet-excited state. Three visible bands in the region 500-600 $\mathrm{nm}$ are the origins of the lowest excited states in different vibrational levels [31]. This absorption feature is comparable with that of the free PPIX in the $\mathrm{PrOH}$ solution, indicating that PPIX has been successfully complexed in the $\mathrm{Ti}(\mathrm{O})$ network. Indeed, the sol-gel process has the advantage of allowing the incorporation of various complexing species such as dyes, redox mediators, providing organic-inorganic composite materials [32-36]. Further evidence came from the XPS measurements (Supporting information-S.2). As an example, the ITO/Ti(O)/ PPIX-FCA electrodes exhibit averaged $\mathrm{Fe} / \mathrm{Ti}$ and PPIX/ Ti ratios of $9.6 \%$ and $1.5 \%$, which are close to those incorporated during the synthesis $(10 \%$ and $1 \%)$.

SEM studies performed on the different gel coated ITO electrodes reveal a similar surface pattern whatever the gel components (Supporting information-S.3) and no welldefined substructure could be observed. The hydrolysiscondensation of $\mathrm{Ti}(\mathrm{OBu})_{4}$ usually results in amorphous $\mathrm{Ti}(\mathrm{O})$ gels, which in contrast to calcinated solids are polymeric compounds having a metal-oxo-based macromolecular network [37].

The electrochemical responses of the $\mathrm{Ti}(\mathrm{O})$ film coated ITO electrode were also studied, as shown in Fig. 4. For an ITO/Ti(O) electrode, a flat potential window ranging from $-0.8 \mathrm{~V}$ to $1.0 \mathrm{~V}$ can be obtained. In the presence of PPIX in the film, an oxidation current wave starting at $0.50 \mathrm{~V}$ was observed in the forward scan only, probably corresponding to the irreversible oxidation of PPIX. In the presence of FCA in the film, a wave around $0.30 \mathrm{~V}$ can be observed, as shown in Fig. 4b. The anodic and cathodic peaks have a potential separation around $90 \mathrm{mV}$, which does not vary much with the potential sweep rate below a value of $0.2 \mathrm{~V} \mathrm{~s}^{-1}$. Analysis of the dependence of the peak currents on the scan rate shows that the peak current is proportional to the square root of the scan rate (Supporting informationS.4). This feature indicates that the oxidation/reduction of FCA inside the $\mathrm{Ti}(\mathrm{O})$ gel is governed by a linear diffusion process instead of a surface process. Free of mobile charges inside, the pristine $\mathrm{Ti}(\mathrm{O})$ film is insulating or poorly conducting in nature. For this reason, the oxidation/reduction of FCA inside the film must be accompanied by a charge compensation step, which is likely to be an ion ingress/egress process considering the present experimental condition. Therefore, the signal displayed in Fig. $4 \mathrm{~b}$ may reflect the linear diffusion of compensating the ion through the film. On the other hand, the diffusion-controlled oxidation of FCA may also originate from an electron hopping mechanism as observed with zirconium oxide gel [36]. Further investigations of this electrochemical response as a function of the gel microstructure and the solution composition are required to fully elucidate this mechanism. Here, we shall mainly focus on the photocurrent response of the $\operatorname{Ti}(\mathrm{O})$ coated electrodes and the important consideration from these voltammetric data is that the available potential window where the gel remains stable ranges from $-0.5 \mathrm{~V}$ to $0.5 \mathrm{~V}$.
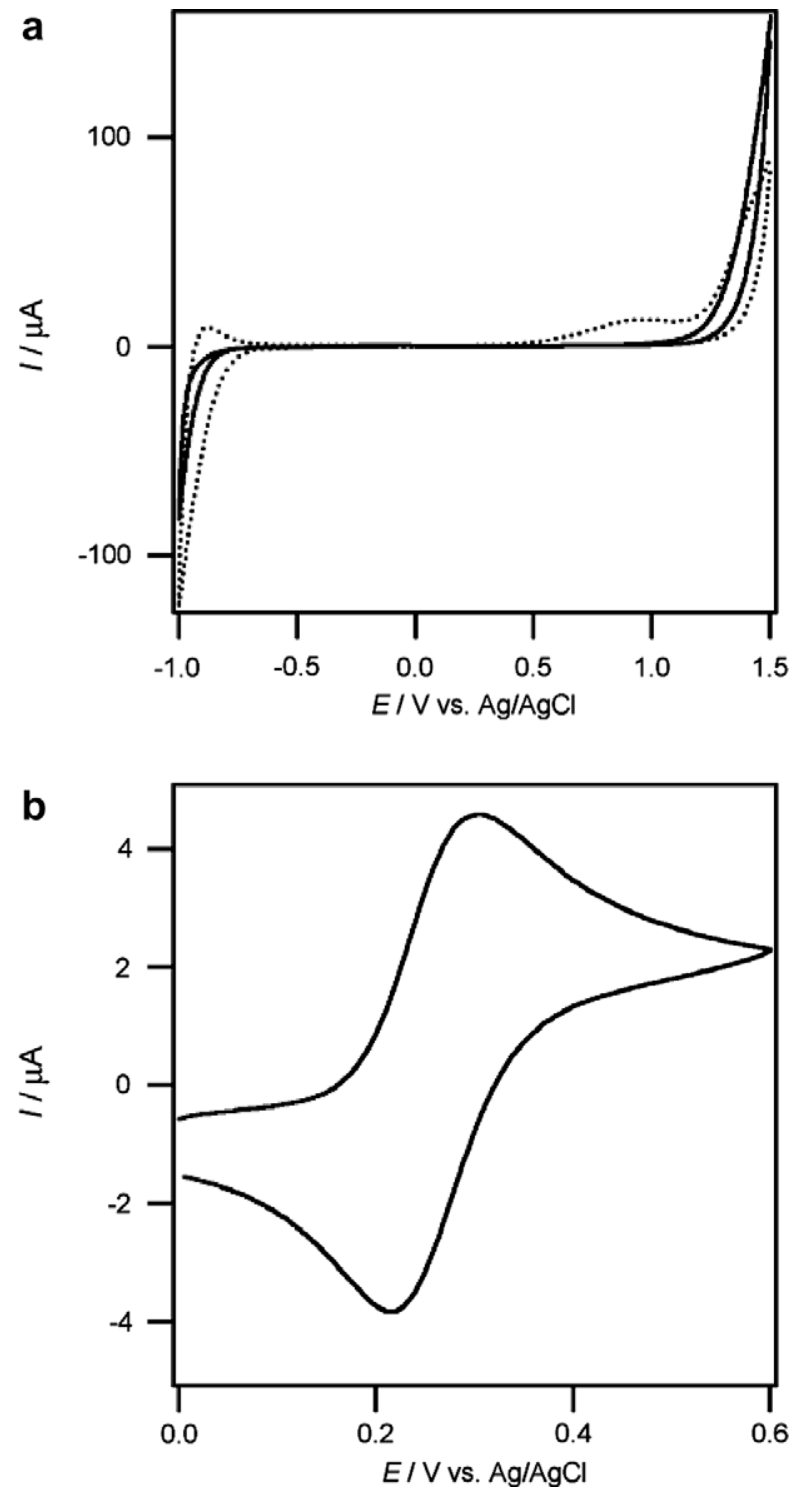

Fig. 4. (a) CVs of the ITO/Ti(O) electrode (solid line) and the ITO/Ti(O)/ PPIX electrode (dotted line); (b) $\mathrm{CV}$ of the ITO/Ti(O)/PPIX-FCA electrode. All the CVs were obtained in the $0.1 \mathrm{M} \mathrm{LiCl}$ solution at a scan rate of $0.05 \mathrm{~V} \mathrm{~s}^{-1}$ (Staircase $\mathrm{CV}$, potential step $5 \mathrm{mV}$ ).

\subsection{Photocurrent responses}

When the photoactive PPIX is incorporated in the Ti(O) gel layer, illumination of the modified ITO electrode onsets the electron transfer reactions at the ITO/Ti(O) film interface. The photoexcited PPIX can be oxidized and/or reduced, depending on the applied electrode potential. Fig. 5a shows the photocurrent transients of an ITO/ $\mathrm{TI}(\mathrm{O}) / \mathrm{PPIX}$ electrode at different applied potentials. Upon illumination, the photocurrent rises slowly to reach a maximum value and then remains more or less constant in the following seconds. When the illumination is stopped, the photocurrent decreases back to zero. The rising feature of photocurrents at short times is either due to the attenuation of the $R C$ component of the cell or to the effect of the fast 

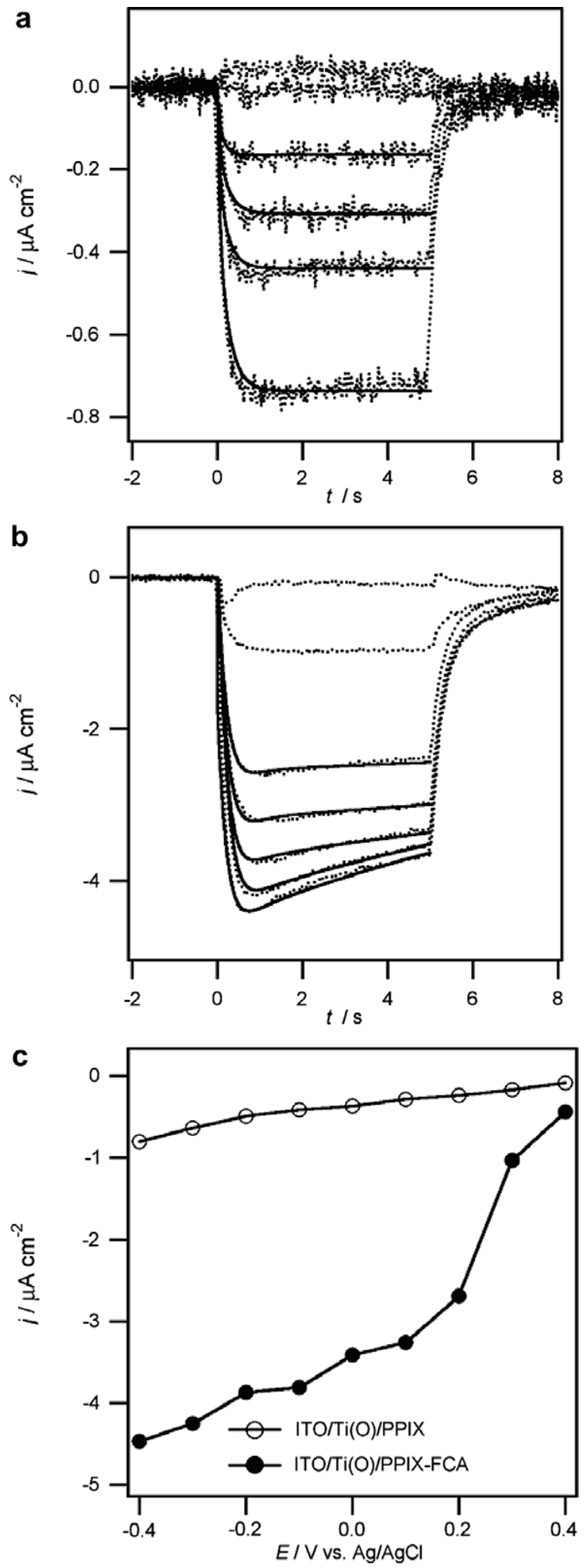

Fig. 5. (a) Photocurrent transients of the ITO/Ti(O)/PPIX electrode at different applied potentials: $-0.40 \mathrm{~V},-0.20 \mathrm{~V}, 0 \mathrm{~V}, 0.20 \mathrm{~V}, 0.40 \mathrm{~V}$ and $0.50 \mathrm{~V}$ from the bottom to top. (b) Photocurrent transients of the ITO/ $\mathrm{Ti}(\mathrm{O}) /$ PPIX-FCA electrode at various applied potentials: $-0.40 \mathrm{~V}$, $-0.30 \mathrm{~V},-0.10 \mathrm{~V}, 0.10 \mathrm{~V}, 0.20 \mathrm{~V}, 0.40 \mathrm{~V}$ and $0.50 \mathrm{~V}$ from the bottom to top. The solid curves are fitted employing Eqs. (14) (a) and (15) (b) and the corresponding fitting parameters are listed in Table 1. (c) Comparison of the maximum photocurrents of the ITO/Ti(O)/PPIX and ITO/Ti(O)/ PPIX-FCA electrodes at various applied potentials. quenching of excited PPIX by electron acceptors present in the system as discussed in Section 3.4. Another feature observed in Fig. 5a is the variation of the photocurrent response with the applied potential. At $0.50 \mathrm{~V}$, a small positive photocurrent can be observed. At potentials more negative than $0.50 \mathrm{~V}$, negative photocurrents arise and the magnitude markedly increases when the potential is tuned to more negative values. Given that the $\mathrm{Ti}(\mathrm{O})$ network is free of mobile ions, the electron transport across the gel film would proceed mostly via electron hopping between the ITO electrode and the PPIX centers. Thus, the electric field inside the $\mathrm{Ti}(\mathrm{O})$ gel layer is a key factor controlling the apparent rate of electron transfer and hence the photocurrent amplitude. Since the PPIX centers are distributed at different distances from the ITO electrode, the observed photocurrent signal corresponds to an integrated response of the PPIX centers located at different levels from the ITO electrode.

To improve the electron hopping within the film, an excess of redox mediator having a carboxylic group, FCA, was complexed in the $\mathrm{Ti}(\mathrm{O})$ network. Displayed in Fig. $5 \mathrm{~b}$ are the photocurrent transient responses obtained at an ITO/Ti(O)/PPIX-FCA electrode at various applied potentials. Two regimes can now be clearly distinguished. At potentials more positive than $0.20 \mathrm{~V}$, the photocurrent magnitude is small. At $0.50 \mathrm{~V}$, the photocurrent transient features a negative initial value and a positive steady-state value, as well as an off-overshoot. At potentials more negative than $0.20 \mathrm{~V}$, the negative photocurrents are enhanced. This can be ascribed to the direct oxidation of FCA, which occurs at $0.20 \mathrm{~V}$ as shown by the CV in Fig. $4 \mathrm{~b}$.

To analyze the role of FCA inside the Ti $(\mathrm{O})$ network, the maximum initial photocurrent observed at ITO/ Ti(O)/PPIX and ITO/Ti(O)/PPIX-FCA electrodes at various applied potentials was compared (Fig. 5c). It is clear that the photocurrent was enhanced in the whole potential range by the presence of FCA. In the potential range from $-0.40 \mathrm{~V}$ to $0.20 \mathrm{~V}$, the photocurrent magnitude was enhanced 5-6 times. The maximum incident photon to current conversion efficiency (IPCE) at $-0.40 \mathrm{~V}$ was increased 5.6 times, from $0.14 \%$ to $0.79 \%$, when FCA was incorporated. The enhancement in the photocurrent magnitude in the presence of FCA can be ascribed to the electron donating and shuttling effects of FCA. That is, FCA can effectively donate an electron to the excited PPIX, by which the correspondingly produced $\mathrm{FCA}^{+}$can be reduced at the ITO electrode. This donating and shuttling process may result in a faster electron transport pathway than the pure electron hopping in the ITO/Ti(O)/PPIX system. This point will be further quantitatively discussed in Section 3.5.

As shown in Fig. 5, the PPIX sensitized $\mathrm{Ti}(\mathrm{O})$ gel layer supported on the ITO electrode preferentially gives rise to negative photocurrents, which necessitates the occurrence of reduction processes at the ITO/film interface. At an ITO/Ti(O)/PPIX electrode, the reduction processes involving the PPIX species can be either the reduction of the excited porphyrin $\left(\right.$ PPIX $\left.^{*}\right)$ or the oxidized porphyrin $\left(\right.$ PPIX $\left.^{+}\right)$: 
PPIX $^{*}+\mathrm{e}^{-} \rightarrow$ PPIX $^{-}$.

PPIX $^{+\cdot}+\mathrm{e}^{-} \rightarrow$ PPIX

$\mathrm{PPIX}^{+} \cdot$ in Eq. (2) can be the product of either the photooxidation of PPIX* at the ITO electrode or the photooxidation of PPIX* by an electron acceptor present in the system. The latter oxidation process not only produces PPIX $^{+}$, the reduction of which (Eq. (2)) gives rise to a cathodic photocurrent, but is also in competition with the oxidation of PPIX ${ }^{*}$ at the ITO electrode resulting in a concomittant anodic photocurrent. This implies that the presence of an electron acceptor in the solution can facilitate the reduction process and depress the oxidation, thus enhancing the cathodic photocurrent. If Eq. (1) is considered, then the electron acceptor can remove electrons from PPIX $^{-\cdot}$ to promote the charge separation, thus accelerating the reduction of PPIX*.

On the other hand, it should be noted here that a concomitant charge transfer reaction at the film/solution interface must take place prior or after the photoreduction process to maintain the electroneutrality of the $\mathrm{Ti}(\mathrm{O})$ film. For example, following the reaction step 1 (Eq. (1)), the produced radical anion PPIX $^{-\cdot}$ can either participate in an electron transfer reaction with an electron acceptor in solution or be compensated by a cation ingress process. These two cases have been schematically illustrated in Fig. 6. In the former case (shown in Fig. 6a), not only the charge separation is accomplished, but also the ground state PPIX is catalytically regenerated. Considering the experimental conditions, dissolved oxygen is the most likely electron acceptor since molecular oxygen has a saturation solubility of $0.25 \mathrm{mM}$ in water [38]. The oxygen effect on the photocurrent response will be further discussed below.

Fig. 6b illustrates the reduction of PPIX*at the ITO electrode followed by a cation ingress process. The situation is in fact similar to the oxidation/reduction of FCA discussed in Section 3.1. The ingress can be that of $\mathrm{Li}^{+}$or $\mathrm{H}^{+}$at a low $\mathrm{pH}$ from the solution to the $\mathrm{Ti}(\mathrm{O})$ film. In this case, however, the PPIX ${ }^{-}$will not be regenerated and correspondingly the ground-state PPIX population will decrease. In the following kinetic analysis, we shall neglect the ion transfer reactions that may take place in order to consider a constant ground-state PPIX population for simplicity.

\subsection{Kinetic modeling}

To rationalize the experimental photocurrent data and understand the kinetics of electron transfer reactions in this photoelectrochemical system, we shall employ the model proposed previously [39]. The overall process can be described by a Latimer type scheme shown in Fig. 7a, in which the photocurrent response is deconvoluted in a series of unit reactions. The occurrence of each reaction depends on the relative energetic levels of the reactants. The corresponding energetic diagram, including the standard redox potentials of reduction and oxidation of PPIX and PPIX*, a

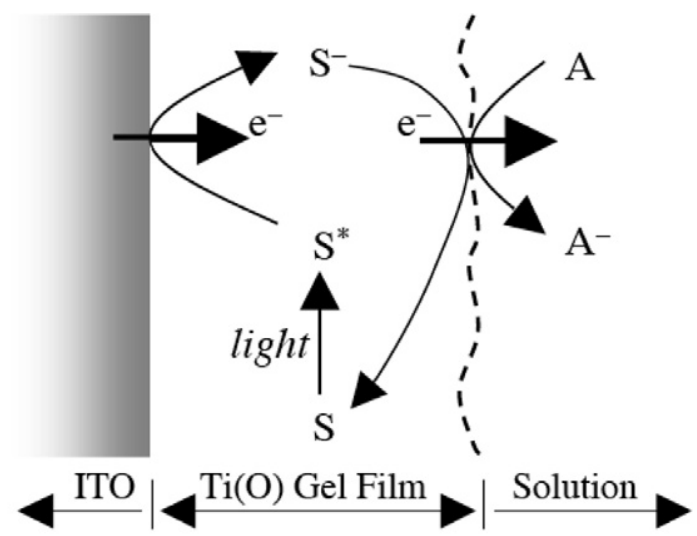

b

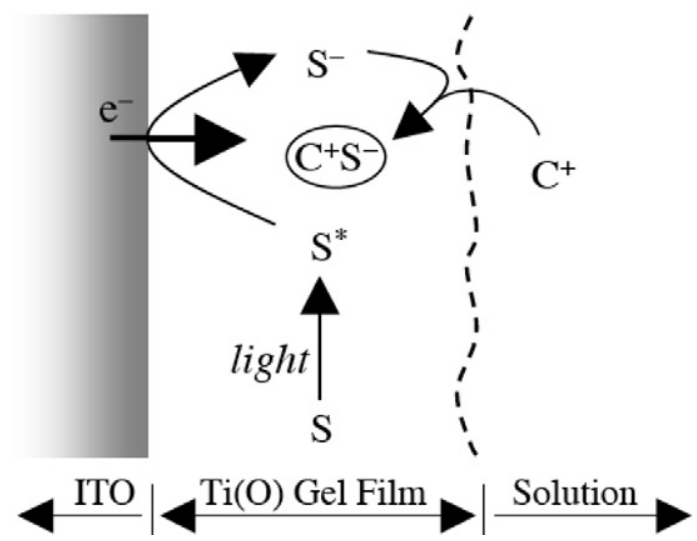

Fig. 6. Schematic illustrations of the reduction of PPIX* at the ITO electrode followed by the catalytic regeneration of PPIX by an electron acceptor (a) or by the ingress of a cation (b) at the film/solution interface $\left(\mathrm{S}^{-}=\right.$PPIX $\left.^{-\cdot}\right)$.

is schematically displayed in Fig. 7b. Since the generation quantum yield of the triplet PPIX ${ }^{*}$ after light absorption is high and the triplet PPIX* has a long lifetime in the millisecond time scale [31], only the triplet excited state is considered. Thus, $E_{\mathrm{S}^{*} / \mathrm{S}^{-}}^{\circ}=0.56 \mathrm{~V}$ and $E_{\mathrm{S}^{+} / \mathrm{S}^{*}}^{\circ}=-0.73 \mathrm{~V}$ in Fig. $7 \mathrm{~b}$ are referred to as the standard redox potentials of reduction and oxidation of the triplet PPIX $^{*}[31,40]$. $E_{\mathrm{FCA}^{+} / \mathrm{FCA}}^{\circ}=0.46 \mathrm{~V}$ (versus $\mathrm{SHE}$ ) was derived from the $\mathrm{CV}$ shown in Fig. 4b. The ion ingress/egress process is also indicated in Fig. 7b. With the help of the energy diagram, the electron transfer reactions possibly occurring in the potential range from $-0.40 \mathrm{~V}$ to $0.20 \mathrm{~V}$ (versus $\mathrm{Ag} / \mathrm{AgCl}$ (sat.), corresponding to about $-0.20 \mathrm{~V}$ to $0.40 \mathrm{~V}$ versus SHE) are also indicated in Fig. $7 b$. The reactions represented by the solid arrows and the dotted arrows give rise to the cathodic and anodic photocurrents, respectively. And those denoted by the dashed arrows correspond to the reactions between the PPIX species and the quenching species present in the system. For simplicity, PPIX is presented as $\mathrm{S}$ and PPIX $^{+}$and PPIX $^{-\cdot}$ are denoted as $\mathrm{S}^{+}$ and $\mathrm{S}^{-}$, respectively.

In terms of the reaction scheme and the energy diagram illustrated in Fig. 7, the initial step of the photoelectrochemical process is the photoexcitation of PPIX denoted as $\mathrm{S}$ : 
a
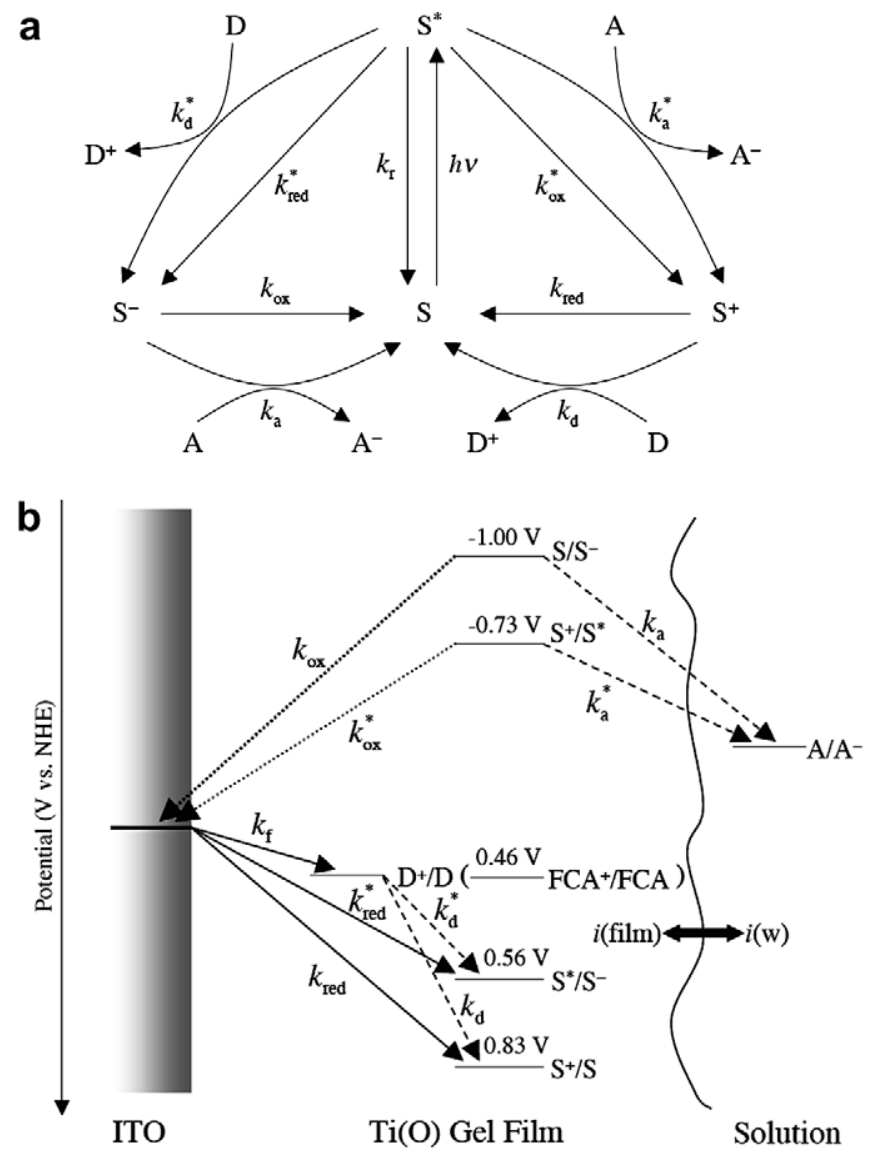

Fig. 7. (a) Schematic representation of the photoinduced electron transfer reactions. S, A and D represent PPIX, electron acceptor and electron donor $\left(\mathrm{S}^{+}=\mathrm{PPIX}^{+}, \mathrm{S}^{-}=\mathrm{PPIX}^{-\cdot}\right)$. (b) The corresponding energetic diagram.

$\mathrm{S} \stackrel{h v}{\rightarrow} \mathbf{S}^{*}$

Here, we let $\mathrm{S}^{*}$ denote the triplet PPIX*. $\mathrm{S}^{*}$ can relax to the ground state through radiative or non-radiative pathways:

$\mathrm{S}^{*} \stackrel{k_{\mathrm{r}}}{\longrightarrow} \mathrm{S}$

$k_{\mathrm{r}}$ represents the rate constant of the intramolecular relaxation of $\mathrm{S}^{*}$. $\mathrm{S}^{*}$ can also be oxidized and reduced directly at the ITO electrode by:

$\mathrm{S}^{*} \stackrel{k_{\mathrm{ox}}^{*}}{\rightarrow} \mathrm{S}^{+}+\mathrm{e}^{-}$

$\mathrm{S}^{*}+\mathrm{e}^{-} \stackrel{k_{\text {red }}^{*}}{\longrightarrow} \mathrm{S}^{-}$

These two electrochemical reactions are simplified as firstorder kinetic processes with rate constants $k_{\mathrm{ox}}^{*}$ and $k_{\mathrm{red}}^{*}$, representing the oxidation and reduction of the triplet PPIX*. Based on the discussion in the last section, $k_{\mathrm{ox}}^{*}$ and $k_{\mathrm{red}}^{*}$ represent the reciprocals of the average time of electron transport through the film. In addition, $\mathrm{S}^{*}$ can also react with the quenching species possibly present in the film or in the solution:

$\mathrm{S}^{*}+\mathrm{A} \stackrel{k_{\mathrm{a}}^{*}}{\rightarrow} \mathrm{S}^{+}+\mathrm{A}^{-}$
$\mathrm{S}^{*}+\mathrm{D} \stackrel{k_{\mathrm{d}}^{*}}{\rightarrow} \mathrm{S}^{-}+\mathrm{D}^{+}$ where $\mathrm{A}$ and $\mathrm{D}$ denote the electron acceptor and electron donor, respectively. Also, these two chemical reactions are considered as first-order kinetic processes for simplicity. Steps 5-8 represent the reactions in which $\mathrm{S}^{*}$ can participate. In these steps, $\mathrm{S}^{+}$and $\mathrm{S}^{-}$are generated, which can be involved in a series of recycling reactions:

$\mathrm{S}^{-} \stackrel{k_{\mathrm{ox}}}{\longrightarrow} \mathrm{S}+\mathrm{e}^{-}$

$\mathrm{S}^{+}+\mathrm{e}^{-\stackrel{k_{\text {red }}}{\longrightarrow} \mathrm{S}}$

$\mathrm{S}^{-}+\mathrm{A} \stackrel{k_{\mathrm{a}}}{\longrightarrow} \mathrm{S}+\mathrm{A}^{-}$

$\mathrm{S}^{+}+\mathrm{D} \stackrel{k_{\mathrm{d}}}{\rightarrow} \mathrm{S}+\mathrm{D}^{+}$

The rate constants of steps 9-12 are different with those of 58. $k_{i}$ and $k_{i}^{*}(i=\mathrm{ox}$, red, a and d) denote the rate constants of reactions involving ground state and excited state PPIX species, respectively. Taking $k_{\mathrm{ox}}$ and $k_{\mathrm{ox}}^{*}$ as an example, they represent the rate constants of oxidation of $\mathrm{S}^{-}$and $\mathrm{S}^{*}$, respectively. Although the driving forces for the oxidations of $\mathrm{S}^{-}$and $\mathrm{S}^{*}$ are different, $k_{\mathrm{ox}}$ and $k_{\mathrm{ox}}^{*}$ should follow the same dependence on the applied potential. For the sake of simplicity, we shall assume $k_{\mathrm{ox}}^{*}=k_{\mathrm{ox}}, k_{\mathrm{red}}^{*}=k_{\mathrm{red}}, k_{\mathrm{a}}^{*}=k_{\mathrm{a}}$ and $k_{\mathrm{d}}^{*}=k_{\mathrm{d}}$ in the simulations of the next several sections.

Furthermore, in the presence of FCA inside the $\mathrm{Ti}(\mathrm{O})$ network, FCA can function as an effective electron donor. However, FCA is considered to be different from other electron donors possibly present in the system as the oxidized FCA mediator, $\mathrm{FCA}^{+}$, can be reduced at the electrode to be regenerated. In this case, FCA functions as the relay, shuttling electron transport between the electrode and the entrapped PPIX. In this case, an additional reaction should be considered in the reaction scheme:

$\mathrm{D}^{+}+\mathrm{e}^{-} \stackrel{k_{\mathrm{f}}}{\rightarrow} \mathrm{D}$

where $k_{\mathrm{f}}$ denotes the rate constant of $\mathrm{FCA}^{+}$reduction.

The above deconvolution of the photoinduced electron transfer process is based on the energy diagram shown in Fig. $7 \mathrm{~b}$, where the electrode potential not only necessarily lies in between $E_{\mathrm{S}^{*} / \mathrm{S}^{-}}^{\circ}$ and $E_{\mathrm{S}^{+}}^{\circ} / \mathrm{S}^{*}$ but also is more negative than $E_{\mathrm{FCA}^{+} / \mathrm{FCA}}^{\circ}$. Otherwise, the reaction scheme should be reconsidered. For example, when the electrode potential is tuned to be more positive than $E^{\circ}{ }_{\mathrm{FCA}^{+} / \mathrm{FCA}}$, the regeneration of $\mathrm{FCA}^{+}$at the electrode is endergonic. Thus, FCA loses its function as the electron transfer relay, although it can still act as an electron donor. Further, when the electrode potential exceeds $E_{\mathrm{S}^{+} / \mathrm{S}^{*}}$, the reduction of $\mathrm{S}^{*}$ at the electrode (step 6) will be largely depressed and correspondingly the cathodic photocurrent will decrease. Either or both of these two situations can explain the marked decrease in the negative photocurrent magnitude in Fig. $5 \mathrm{~b}$ when the electrode potential exceeds $0.30 \mathrm{~V}$ (versus $\mathrm{Ag} / \mathrm{AgCl}$ ), as well as the appearance of anodic photocurrents at the more positive potentials. Since most of the photocurrent measurements were carried out in the potential range below $0.30 \mathrm{~V}$, we shall only consider the energy scheme shown in Fig. $7 \mathrm{~b}$ in the following discussion. 


\subsection{Kinetic analysis of non-mediated photocurrents}

On the basis of the model presented in Section 3.3 and considering the ITO/film interface, mathematical development (Appendix A) yields the photocurrent for the ITO/ $\mathrm{Ti}(\mathrm{O}) / \mathrm{PPIX}$ electrode (named non-mediated photocurrent as FCA is absent) which can be considered as a sum of the steady-state photocurrent (time-independent) and transient photocurrent (time dependent):

$$
\begin{aligned}
j_{\mathrm{ph}} & =j_{\mathrm{ph}}^{\mathrm{ss}}+j_{\mathrm{ph}}(t) \\
& =g \alpha+g\left[\beta \mathrm{e}^{-\left(k_{\mathrm{ox}}+k_{\mathrm{a}}\right) t}+\gamma \mathrm{e}^{-\left(k_{\mathrm{red}}+k_{\mathrm{d}}\right) t}+\chi \mathrm{e}^{(-t / R C)}\right],
\end{aligned}
$$

where $j_{\mathrm{ph}}^{\mathrm{ss}}$ and $j_{\mathrm{ph}}(t)$ denote the steady-state and transient photocurrents, respectively. $j_{\mathrm{ph}}(t)$ has three contributions as indicated by the three terms in the bracket, which represent the oxidation, reduction and charging photocurrent transients, respectively. $g$ is a parameter determined by the light absorption of the film and the incident light intensity. $\alpha, \beta, \gamma$ and $\chi$ are kinetic parameters including $k_{i}(i=$ ox, red, a and d) and the $R C$ component as defined in Appendix A.

First, the influence of the kinetic parameters included in Eq. (14) except $k_{\mathrm{d}}$ on the photocurrent transient was examined theoretically. The parameter $k_{\mathrm{d}}$ will be discussed in Section 3.5. Shown in Fig. 8 are the calculated influences of the parameters $k_{\mathrm{ox}}, k_{\mathrm{red}}, k_{\mathrm{a}}$ and $R C$. These calculations were obtained by taking $g=560 \mu \mathrm{A} \mathrm{cm}^{-2}, \quad k_{\mathrm{r}}=1800 \mathrm{~s}^{-1}$, $k_{\mathrm{ox}}=2.0 \mathrm{~s}^{-1}, k_{\mathrm{red}}=2.8 \mathrm{~s}^{-1}, k_{\mathrm{a}}=1.2 \mathrm{~s}^{-1}, k_{\mathrm{d}}=1.0 \mathrm{~s}^{-1}$ and $R C=0.05 \mathrm{~s}$ unless specified otherwise in the figure. This set of values generates a theoretical photocurrent transient close to what is observed experimentally. $g=560 \mu \mathrm{A} \mathrm{cm} \mathrm{cm}^{-2}$ was calculated from the light absorption of the film and the incident light intensity (Eq. (A.9) in Appendix A) and $k_{\mathrm{r}}=1800 \mathrm{~s}^{-1}$ was taken from Ref. [27]. Fig. 8d illustrates that the $R C$ component only slightly attenuates the initial
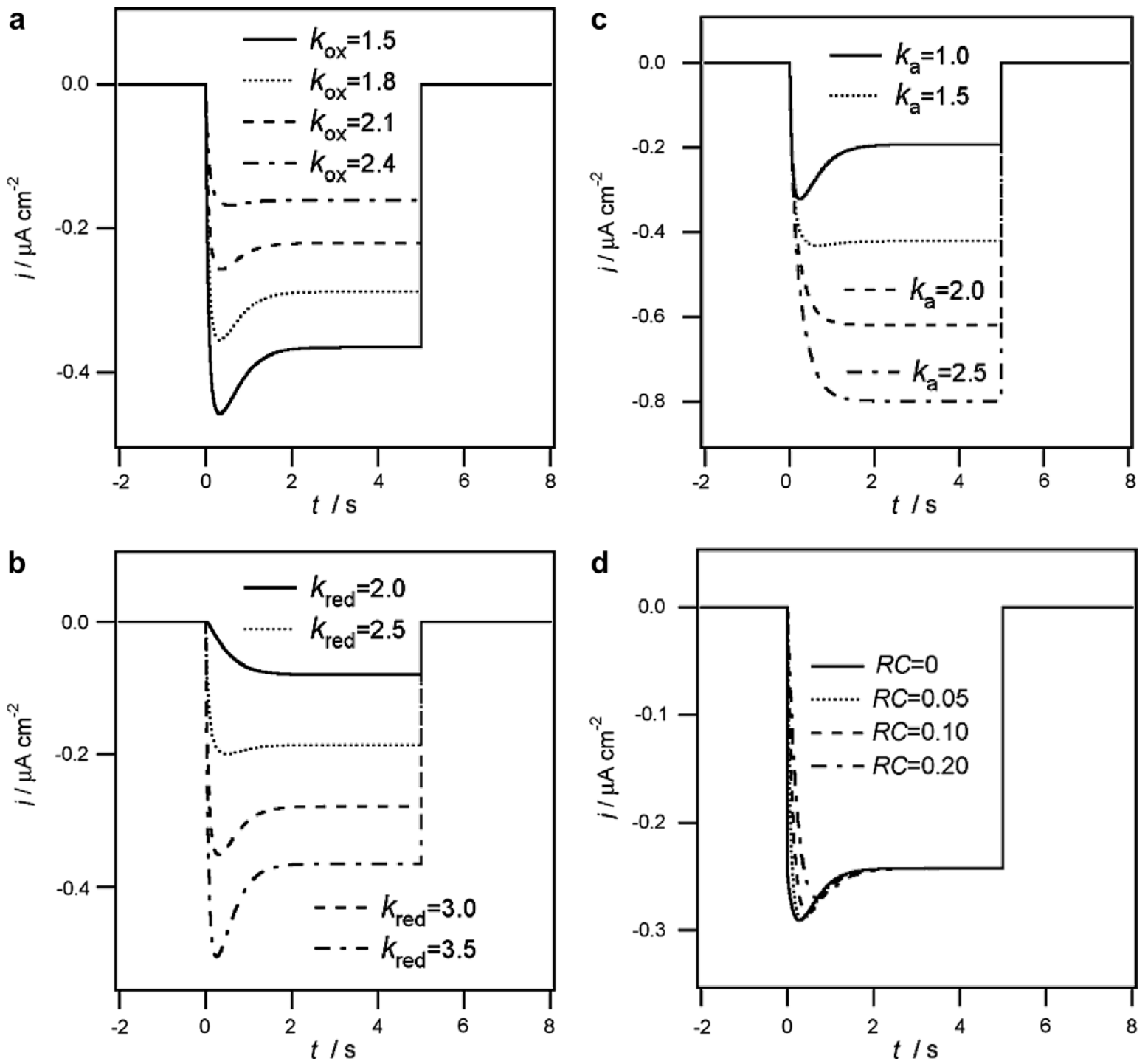

Fig. 8. Evaluations of the effect of $k_{\mathrm{ox}}, k_{\mathrm{red}}, k_{\mathrm{a}}$ and $R C$ on the photocurrent transients according to Eq. (14). The parameters employed are as follows unless specified otherwise in the figures: $g=560 \mu \mathrm{A} \mathrm{cm} \mathrm{c}^{-2}, k_{\mathrm{r}}=1800 \mathrm{~s}^{-1}, k_{\mathrm{ox}}=2.0 \mathrm{~s}^{-1}, k_{\mathrm{red}}=2.8 \mathrm{~s}^{-1}, k_{\mathrm{a}}=1.2 \mathrm{~s}^{-1}, k_{\mathrm{d}}=1.0 \mathrm{~s}^{-1}$ and $R C=0.05 \mathrm{~s}$. 
part of the photocurrent and affects its shape, but it does not influence the steady-state photocurrent. The other three parameters, $k_{\mathrm{ox}}, k_{\mathrm{red}}$ and $k_{\mathrm{a}}$, convolute together to determine the photocurrent magnitude and it is difficult to resolve their respective contributions to the observed photocurrent. However, at a constant $k_{\mathrm{a}}$ value, the relative magnitude of $k_{\mathrm{ox}}$ and $k_{\text {red }}$ determines the shape of the initial part of the photocurrent transient, as shown in Fig. 8a and b. For example, if $k_{\text {red }}$ is much larger than $k_{\mathrm{ox}}$, then the initial photocurrent rising is sharp, which is followed by a decay at short times and a steady state at longer times. In contrast, when $k_{\text {red }}$ is comparable with or smaller than $k_{\mathrm{ox}}$, the photocurrent rises slowly and then reachs a steady state directly at longer times.

The large number of parameters in Eq. (14) prevents an accurate fitting of the experimental data. It is necessary to limit the number of adjustable parameters to extract kinetic information from the fitting. Therefore, we shall start with the simplest experimental system, the ITO/Ti(O)/PPIX electrode in the absence of oxygen in the solution. The photocurrent transient responses of this system are shown in Fig. 9a. In comparison with the photocurrent responses displayed in Fig. 5a where oxygen is present in the solution, a strong effect of solvated oxygen can be found. The removal of oxygen in the solution results in the depression of photocurrent magnitude, as compared in Fig. 9b. This confirms the electron accepting nature of the dissolved oxygen in the solution. Thus, in the absence of oxygen in the solution, the consideration of $k_{\mathrm{a}}$ can be simplified since the influence of oxygen can be neglected. For $k_{\mathrm{ox}}$ and $k_{\mathrm{red}}$, since they denote the oxidation and reduction of PPIX species at the ITO electrode, they should be dependent on the applied potential. Furthermore, $k_{\text {ox }}$ and $k_{\text {red }}$ should be exempted from the influence of the electron acceptor and donor present in the system. That is, at a certain applied potential, $k_{\mathrm{ox}}$ and $k_{\text {red }}$ are the same for the different experimental conditions. A general comparison between the simulations with the experimental curves shown in Fig. 9a indicates that $k_{\mathrm{a}}=1.2 \mathrm{~s}^{-1}, k_{\mathrm{d}}=1.0 \mathrm{~s}^{-1}$ and $R C=0.05 \mathrm{~s}$. $k_{\mathrm{a}}$ cannot be assigned as 0 , which means that except the dissolved oxygen some other species can also function as the electron acceptor. Possible candidates might be the $\mathrm{Ti}^{4+}$ site in the gel network [41] and the water molecule. $k_{\mathrm{d}}$ is assigned as 1.0 to fit all the experimental curves but the chemical nature of the electron donor has not been elucidated, however, only the gel matrix and/or solvent can play this role considering the experimental conditions. This electron donor is designated "intrinsic electron donor" to discriminate later with FCA present in the ITO/Ti(O)/ PPIX-FCA electrode.

Fittings of the photocurrent transients employing Eq. (14) are shown as dashed curves in Fig. 9a. It can be seen that Eq. (14) provides an adequate description of the photocurrent data. The fitting also provides information to $k_{\mathrm{ox}}$ and $k_{\text {red }}$, whose values at various applied potentials are summarized in Table 1 . Using the $k_{\mathrm{ox}}$ and $k_{\mathrm{red}}$ values obtained above, the photocurrent responses of the ITO/ $\mathrm{Ti}(\mathrm{O}) / \mathrm{PPIX}$ electrode in air saturated solution were also fit-
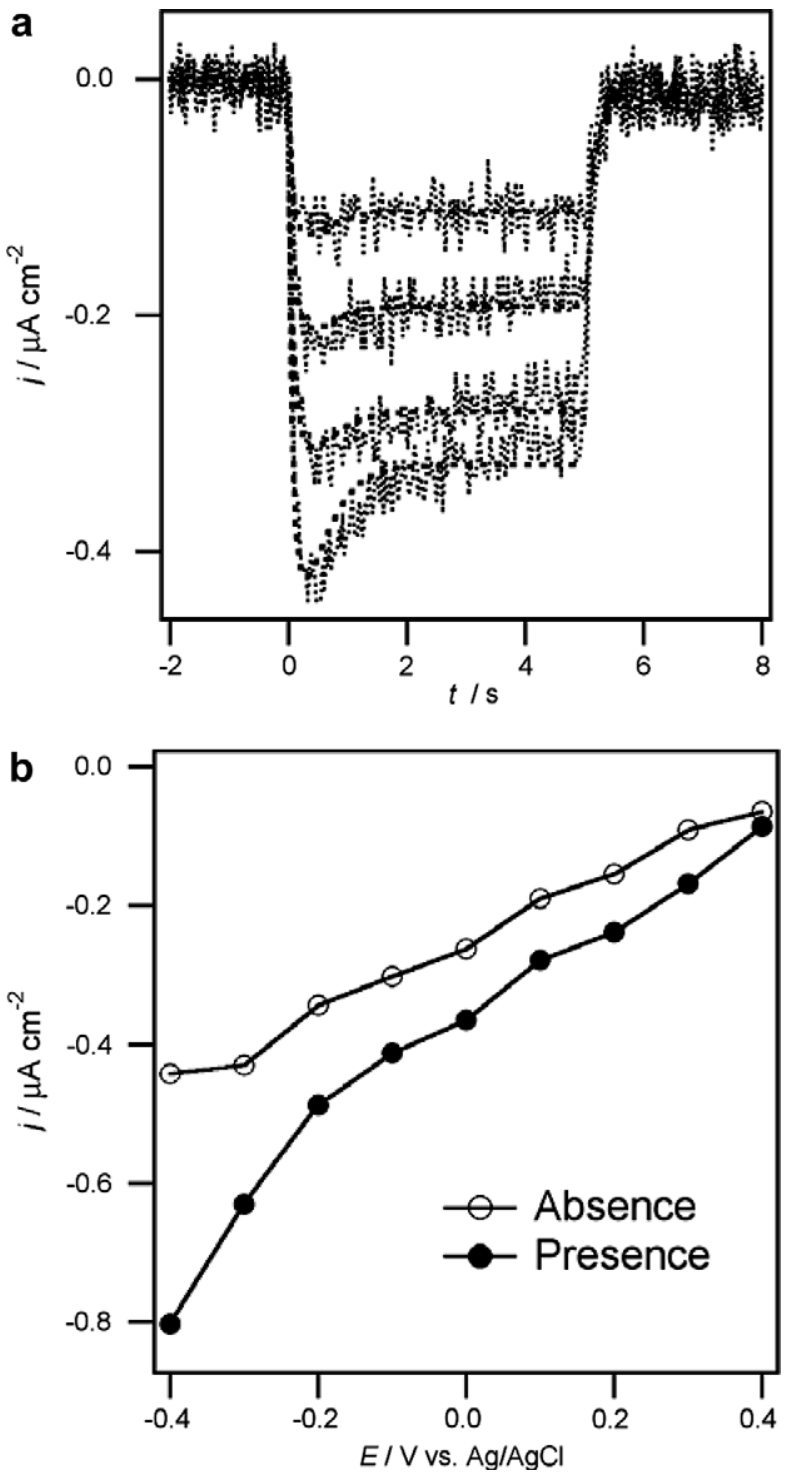

Fig. 9. (a) Photocurrent transients of the ITO/Ti(O)/PPIX electrode at various applied potentials in the absence of oxygen. The potentials are $-0.40 \mathrm{~V},-0.20 \mathrm{~V}, 0 \mathrm{~V}$ and $0.20 \mathrm{~V}$ from the bottom to top. The dashed lines are fitting employing Eq. (14) and the corresponding fitted parameters are listed in Table 1. (b) Comparison of the maximum negative photocurrents of the ITO/Ti(O)/PPIX electrode in the absence and presence of oxygen at various applied potentials.

Table 1

The values for various rate constants obtained by fitting the photocurrent data using Eqs. (14) and (15)

\begin{tabular}{llllllll}
\hline $\begin{array}{l}E_{\text {app }} \\
(\mathrm{V})\end{array}$ & $\begin{array}{l}k_{\mathrm{ox}} \\
\left(\mathrm{s}^{-1}\right)\end{array}$ & $\begin{array}{l}k_{\mathrm{red}} \\
\left(\mathrm{s}^{-1}\right)\end{array}$ & $\begin{array}{l}k_{\mathrm{a}}^{\mathrm{noO}} \mathrm{s}^{-1} \\
\left(\mathrm{~s}^{-1}\right)\end{array}$ & $\begin{array}{l}k_{\mathrm{a}}^{\mathrm{O}_{2}} \\
\left(\mathrm{~s}^{-1}\right)\end{array}$ & $\begin{array}{l}k_{\mathrm{d}}^{\mathrm{noFC}} \\
\left(\mathrm{s}^{-1}\right)\end{array}$ & $\begin{array}{l}k_{\mathrm{d}}^{\mathrm{FC}} \\
\left(\mathrm{s}^{-1}\right)\end{array}$ & $\begin{array}{l}k_{\mathrm{f}} \\
\left(\mathrm{s}^{-1}\right)\end{array}$ \\
\hline-0.40 & 1.87 & 3.08 & 1.20 & 2.17 & 1.00 & 5.30 & 0.093 \\
-0.30 & 1.92 & 2.95 & 1.20 & 1.83 & 1.00 & 5.16 & 0.074 \\
-0.20 & 2.02 & 2.83 & 1.20 & 1.65 & 1.00 & 4.98 & 0.046 \\
-0.10 & 2.12 & 2.81 & 1.20 & 1.52 & 1.00 & 4.75 & 0.038 \\
0 & 2.21 & 2.76 & 1.20 & 1.47 & 1.00 & 4.32 & 0.026 \\
0.10 & 2.34 & 2.66 & 1.20 & 1.34 & 1.00 & 4.22 & 0.022 \\
0.20 & 2.40 & 2.55 & 1.20 & 1.22 & 1.00 & 4.11 & 0.016 \\
\hline
\end{tabular}

$k_{\mathrm{a}}^{\mathrm{noO}}{ }_{2}$ and $k_{\mathrm{a}}^{\mathrm{O}_{2}}$ represent $k_{\mathrm{a}}$ in the absence and presence of oxygen. $k_{\mathrm{d}}^{\mathrm{noFC}}$ and $k_{\mathrm{d}}^{\mathrm{FC}}$ denote $k_{\mathrm{d}}$ in the absence and presence of FCA in the gel. 
ted, as shown by the solid curves in Fig. 5a. In these fittings, $k_{\mathrm{d}}$ was kept to be 1.00 and only $k_{\mathrm{a}}$ was left as the fitting parameter. The obtained $k_{\mathrm{a}}$ values at various applied potentials are also listed in Table 1 . It is apparent that $k_{\mathrm{a}}$ was increased in the presence of oxygen, confirming quantitatively that the dissolved oxygen functions as an effective electron acceptor. Furthermore, in the presence of oxygen, $k_{\mathrm{a}}$ is not constant anymore but decreases when the applied potential is more positive. The dependence of $k_{\mathrm{ox}}, k_{\mathrm{red}}$ and $k_{\mathrm{a}}$ on the electrode potential will be further discussed in Section 3.6.

\subsection{Kinetic analysis of mediated photocurrents}

In the presence of FCA inside the $\operatorname{Ti}(\mathrm{O})$ structure, the photocurrent called mediated photocurrent here originates from steps 5, 6, 9, 10 and 13. The mathematical expression of the photocurrent (Appendix B) is:

$$
\begin{aligned}
j_{\mathrm{ph}}= & j_{\mathrm{ph}}^{\mathrm{ss}}+j_{\mathrm{ph}}(t) \\
= & g \delta+g\left[\phi \mathrm{e}^{-\left(k_{\mathrm{ox}}+k_{\mathrm{a}}\right) t}+\rho \mathrm{e}^{-\left(k_{\mathrm{red}}+k_{\mathrm{d}}\right) t}+\xi \mathrm{e}^{-k_{\mathrm{f}} t}\right. \\
& \left.+\zeta \mathrm{e}^{(-t / R C)}\right]
\end{aligned}
$$

where $j_{\mathrm{ph}}(t)$ has four components, one more due to the incorporation of FCA. $\delta, \phi, \rho, \xi$ and $\zeta$ are kinetic parameters including $k_{i}(i=\mathrm{ox}, \mathrm{red}, \mathrm{a}, \mathrm{d}$ and $\mathrm{f})$ and the $R C$ component as defined in Appendix B. $k_{\mathrm{f}}$ is a new parameter present in the system and $k_{\mathrm{d}}$ is also expected to change due to the presence of FCA in the system. The influence of these two parameters on the photocurrent transient was first examined, as shown in Fig. 10. The calculations were obtained using $g=560 \mu \mathrm{A} \mathrm{cm}^{-2}, \quad k_{\mathrm{r}}=1800 \mathrm{~s}^{-1}$, $k_{\mathrm{ox}}=2.0 \mathrm{~s}^{-1}, \quad k_{\mathrm{red}}=2.8 \mathrm{~s}^{-1}, \quad k_{\mathrm{a}}=1.2 \mathrm{~s}^{-1}, \quad k_{\mathrm{d}}=5.0 \mathrm{~s}^{-1}$, $k_{\mathrm{f}}=0.05 \mathrm{~s}^{-1}$ and $R C=0.25 \mathrm{~s}$ unless specified otherwise in the figure. Comparing the influence of $k_{\mathrm{d}}$ and $k_{\mathrm{f}}$ on the photocurrent response indicates that the initial photocurrent magnitude is mainly controlled by the parameter $k_{\mathrm{d}}$ whereas $k_{\mathrm{f}}$ only determines the feature of the photocurrent transient at longer times. As shown in Fig. 10a, the larger the $k_{\mathrm{d}}$ the larger the initial photocurrent. From Fig. 10b, it is apparent that $k_{\mathrm{f}}$ has little effect on the photocurrent at shorter times and increasing $k_{\mathrm{f}}$ only results in a slight decrease of the initial photocurrent. Under this condition, the fitting of the photocurrent responses of the ITO/Ti(O)/ PPIX-FCA electrode becomes easy. As displayed in Fig. 5b, the solid curves correspond to the fittings using Eq. (15). The fitting of experimental photocurrent transients also provides information to $k_{\mathrm{d}}$ and $k_{\mathrm{f}}$, which are summarized in Table 1.

\subsection{Dependence of the rate constants on the applied potential}

The fittings of experimental photocurrent transients in Section 3.4 result in $k_{\mathrm{ox}}, k_{\mathrm{red}}$ and $k_{\mathrm{a}}$ values at various applied potentials. And those in Section 3.5 give $k_{\mathrm{d}}$ and $k_{\mathrm{f}}$ values. All these values can be found in Table 1 . Fig. 11 illustrates the exponential dependence of $k_{\mathrm{ox}}, k_{\mathrm{red}}$
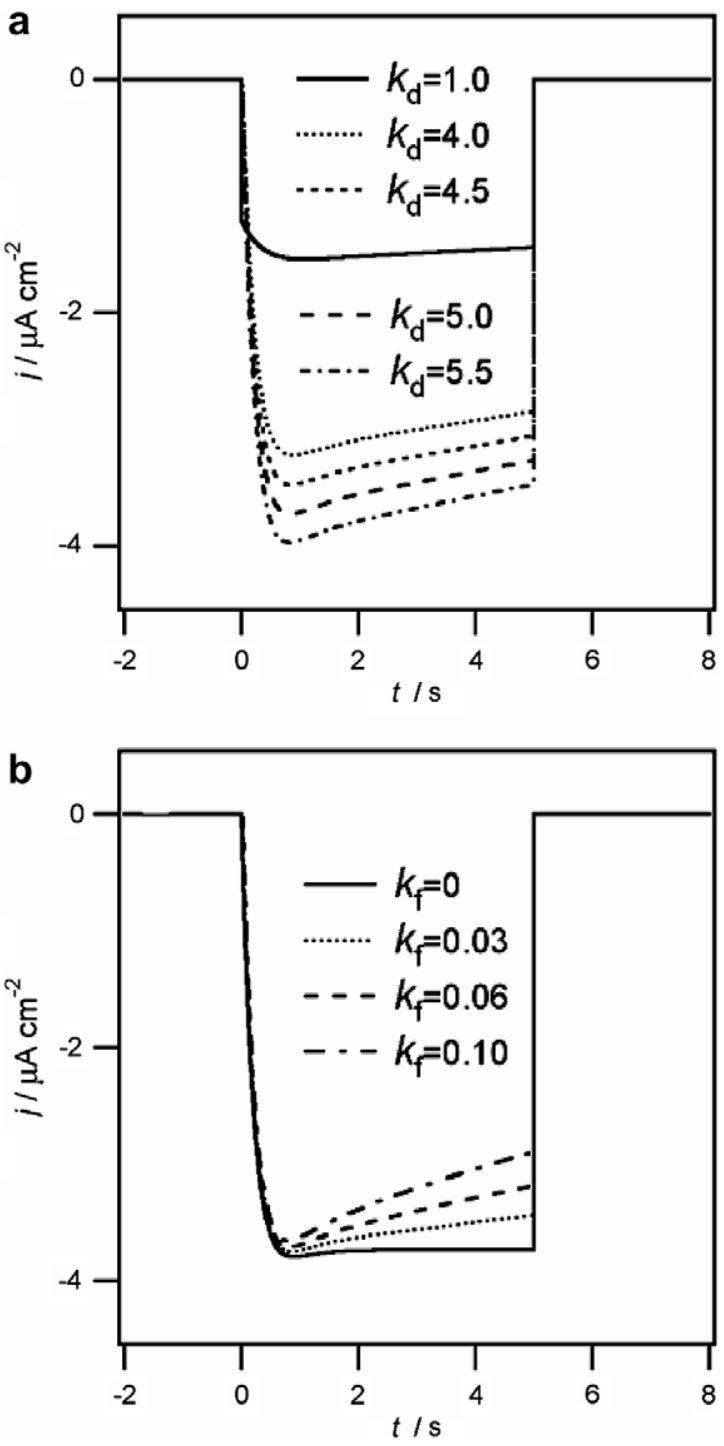

Fig. 10. Evaluations of the effect of $k_{\mathrm{d}}$ and $k_{\mathrm{f}}$ on the photocurrent transients according to Eq. (15). The parameters employed are as follows unless specified otherwise in the figures: $g=560 \mu \mathrm{A} \mathrm{cm}{ }^{-2}, k_{\mathrm{r}}=1800 \mathrm{~s}^{-1}$, $k_{\mathrm{ox}}=2.0 \mathrm{~s}^{-1}, k_{\mathrm{red}}=2.8 \mathrm{~s}^{-1}, k_{\mathrm{a}}=1.2 \mathrm{~s}^{-1}, k_{\mathrm{d}}=5.0 \mathrm{~s}^{-1}, k_{\mathrm{f}}=0.05 \mathrm{~s}^{-1}$, and $R C=0.25 \mathrm{~s}$.

and $k_{\mathrm{a}}$ on the applied potential. Positively increasing the applied potential results in an increase of $k_{\mathrm{ox}}$ and a decrease of $k_{\text {red }}$. The shift of the electrode potential alters its relative position with respect to the standard reduction and oxidation potentials of the PPIX species, accordingly, the driving forces for the reduction and oxidation processes. On the other hand, the oxidation and reduction of PPIX species at the ITO electrode are not that fast since $k_{\text {ox }}$ and $k_{\text {red }}$ values are small as shown in Table 1 . The fittings of $\log k_{\mathrm{ox}}$ and $\log k_{\text {red }}$ versus the applied potential give the electron transfer coefficients of 0.012 and 0.007 for the oxidation and reduction processes, respectively, which indicates that only a small portion of the applied potential operates on the electron transfer process. This can be ascribed to the non-conducting nature of the $\mathrm{Ti}(\mathrm{O})$ network. 


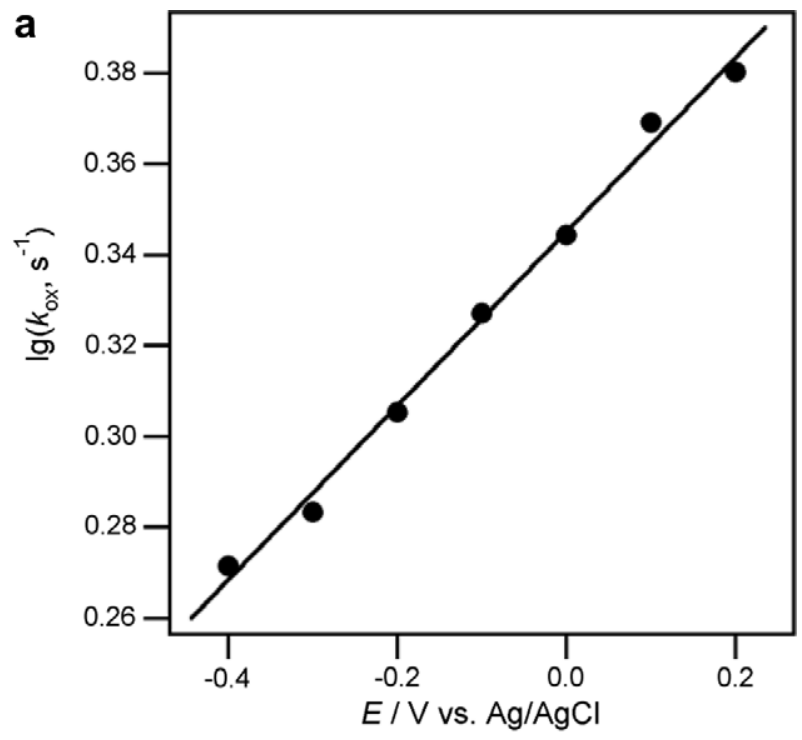

As demonstrated in Section 3.4, $k_{\mathrm{a}}$ remains constant in the absence of oxygen. But in the presence of oxygen, $k_{\mathrm{a}}$ becomes potential dependent, as illustrated in Fig. 11c. $k_{\mathrm{a}}$ represents the pseudo-first order rate constant of electron extraction from PPIX species by dissolved oxygen, which occurs at the film/solution interface. The potential dependence of $k_{\mathrm{a}}$ maybe associated with the potential effect on the proton distribution in the diffuse layer. It is well known that in an aqueous solution, the proton is usually involved in the oxygen reduction process, which has also been investigated in the next section. Considering that the photocurrent data shown in Fig. 5a were obtained in a neutral $(\mathrm{pH}$ 7.0) aqueous medium, the potential variation may alter the proton profile in the diffuse layer to affect the oxygen reduction.
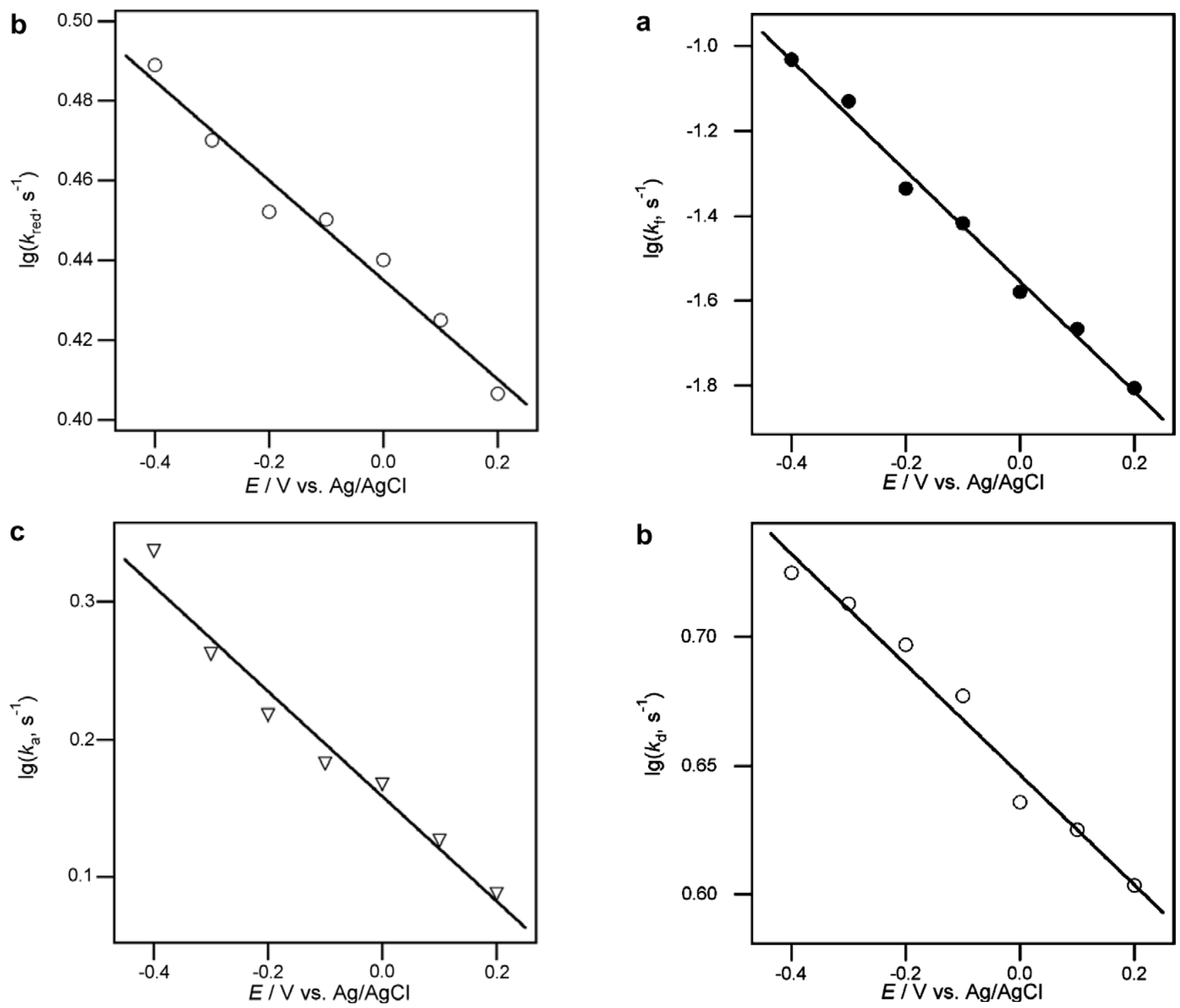

Fig. 11. Rate constants $k_{\text {ox }}$ (a), $k_{\text {red }}$ (b) and $k_{\mathrm{a}}$ (c) as a function of the applied potentials. The values were extracted from the fitting photocurrent transients in Figs. 5a and 9 using Eq. (14).

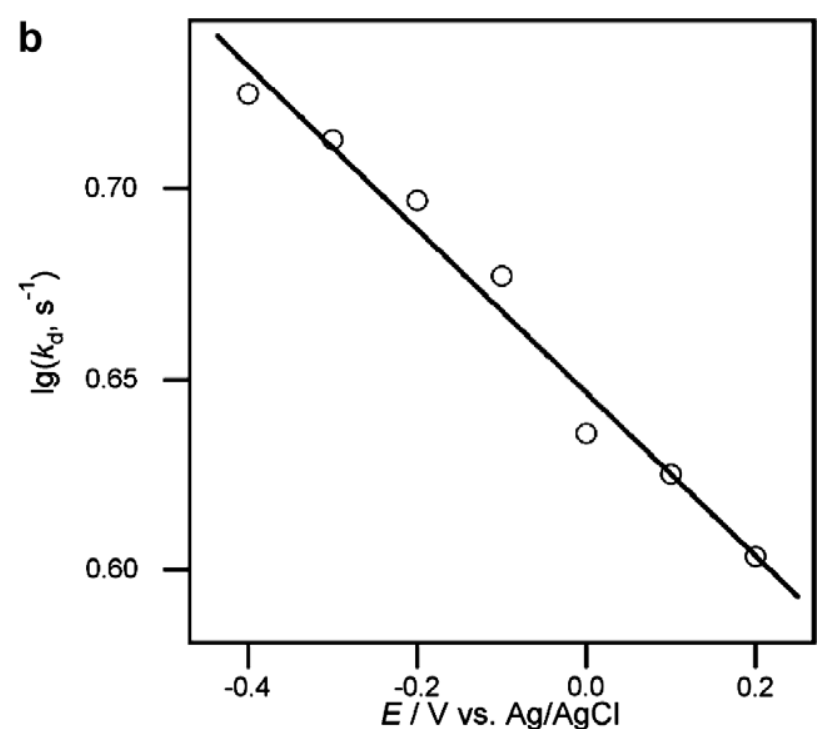

Fig. 12. Rate constants $k_{\mathrm{f}}$ (a) and $k_{\mathrm{d}}$ (b) as a function of the applied potential. The values were extracted from the fitting photocurrent data shown in Fig. 5b using Eq. (15). 
The dependences of $k_{\mathrm{d}}$ and $k_{\mathrm{f}}$ derived from the fittings in Section 3.5 on the applied potential are also displayed in Fig. 12. It is clear that $k_{\mathrm{f}}$ shows an exponential dependence on the applied potential, decreasing when the applied potential is more positive. Since $k_{\mathrm{f}}$ denotes the rate constant of $\mathrm{FCA}^{+}$reduction at the ITO electrode, the positive shift of electrode potential with respect to $E_{\mathrm{FCA}^{+} / \mathrm{FCA}}^{\circ}$ increases the driving force associated with $k_{\mathrm{f}}$. The fitting of $\log k_{\mathrm{f}}$ with respect to the applied potential yielded an electron transfer coefficient of 0.078 , which is much larger than those obtained above. This could be due to the higher quantity of FCA present in the gel, 10 times higher than PPIX. For $k_{\mathrm{d}}$, as shown in Fig. $12 \mathrm{~b}$, it also displays an exponential dependence on the applied potential. $k_{\mathrm{d}}$ denotes the pseudo-first order rate constant of electron donation to the PPIX species. Since both FCA and PPIX are anchored to the $\operatorname{Ti}(\mathrm{O})$ matrix, the electron transfer between them only occurs by hopping, which certainly depends on the electric field inside the film. This situation is different with the electron quenching of PPIX by dissolved oxygen, which occurs at the film/solution interface and the diffusion of oxygen in the solution and/or in the $\mathrm{Ti}(\mathrm{O})$ network pores plays a role. The fitting of $\log k_{\mathrm{d}}$ with respect to the applied potential yielded an electron transfer coefficient of 0.013 , which is close to those of PPIX oxidation and reduction processes.

\subsection{Oxygen and $p H$ effect}

The presence of oxygen in the solution results in a larger photocurrent. Indeed, the oxygen quenching of photoexcited porphyrin is well known $[31,42]$. In aqueous media, the oxygen reduction is a multielectron reaction, either two-electron reduction to give peroxide or four-electron reduction to produce water. The medium $\mathrm{pH}$ also plays an important role in the oxygen reduction process [43]. Therefore, the influence of $\mathrm{pH}$ on the photocurrent response was investigated. The dotted curves in Fig. 13a correspond to the photocurrent transients of the ITO/ $\mathrm{Ti}(\mathrm{O}) / \mathrm{PPIX}-\mathrm{FCA}$ electrode at $-0.40 \mathrm{~V}$ at three $\mathrm{pH}$. A strong effect of $\mathrm{pH}$ on the photocurrent magnitude was observed. The photocurrent at $\mathrm{pH} 4.0$ is more than twice that at $\mathrm{pH} 9.0$ (however, at very alkaline $\mathrm{pHs}$ the stability of the xerogel and/or dye and electron mediator leakage [44] may interfere). The current increment by lowering $\mathrm{pH}$ was observed in the potential range lower than $0.30 \mathrm{~V}$, as shown in Fig. 13b. The solid curves in Fig. 13a represent the fittings employing Eq. (15). Assuming the variation in $\mathrm{pH}$ only alters $k_{\mathrm{a}}$, all other parameters used in the fittings are the same as those employed to fit the photocurrent transient at $-0.40 \mathrm{~V}$ at the neutral condition, that is, $g=560 \mu \mathrm{A} \mathrm{cm}^{-2}, k_{\mathrm{r}}=1800 \mathrm{~s}^{-1}, k_{\mathrm{ox}}=1.87 \mathrm{~s}^{-1}$, $k_{\text {red }}=3.08 \mathrm{~s}^{-1}, \quad k_{\mathrm{d}}=5.30 \mathrm{~s}^{-1}, \quad k_{\mathrm{f}}=0.093 \mathrm{~s}^{-1}$ and $R C=$ $0.25 \mathrm{~s}$. The fittings provide us $k_{\mathrm{a}}$ values of $5.35,3.10$ and 0.42 for $\mathrm{pH}$ at $4.0,6.0$ and 9.0, respectively. Obviously, $k_{\mathrm{a}}$ decreases sharply with increasing the solution $\mathrm{pH}$. However, it should be mentioned that protons might also func- tion as the electron acceptor, especially at very acidic conditions. Therefore, the variation of $k_{\mathrm{a}}$ with $\mathrm{pH}$ can be ascribed to the convolution of the $\mathrm{pH}$ dependent oxygen reduction and the proton reduction. Whether they occur or not, the devolution of these two processes if both occur requires careful control of the experimental conditions.

The influence of $\mathrm{pH}$ on the photocurrent response was further studied in the case of absence of oxygen. As shown in Section 3.4, when oxygen is absent in the system, $k_{\mathrm{a}}$ can be kept constant. Under this condition, unless the reaction involving this intrinsic electron acceptor is also $\mathrm{pH}$ dependent, the photocurrent should be invariant with solution $\mathrm{pH}$. As shown in Fig. 14, the photocurrent responses of the ITO/Ti(O)/PPIX-FCA electrode in the absence of
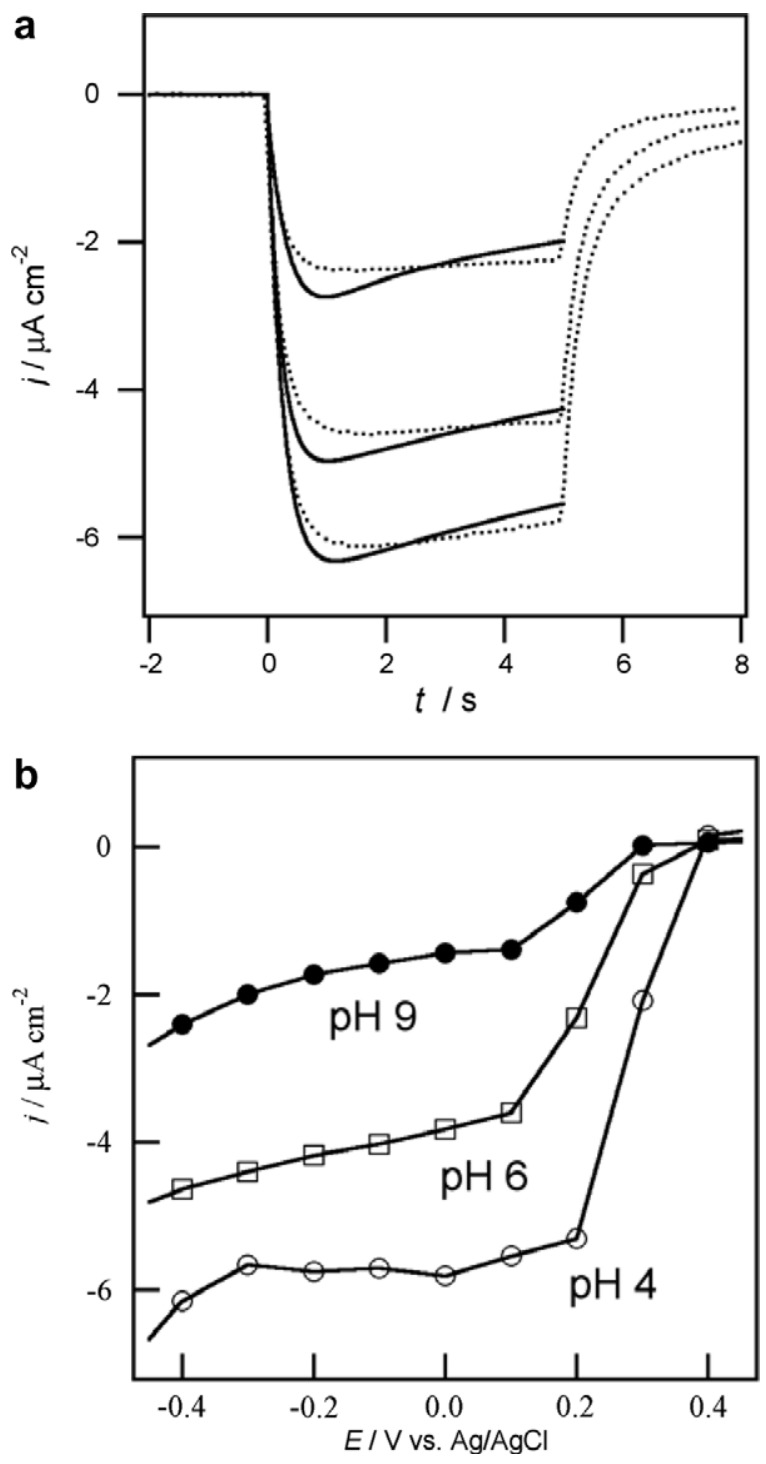

Fig. 13. (a) The influence of solution $\mathrm{pH}$ on the photocurrent transient of the ITO/Ti(O)/PPIX-FCA electrode at $-0.40 \mathrm{~V}$. The $\mathrm{pH}$ is 4,6 and 9 from the bottom to top.The solid lines are the fittings using Eq. (15) with $g=560 \mu \mathrm{A} \mathrm{cm}^{-2}, \quad k_{\mathrm{r}}=1800 \mathrm{~s}^{-1}, \quad k_{\mathrm{ox}}=1.87 \mathrm{~s}^{-1}, \quad k_{\mathrm{red}}=3.08 \mathrm{~s}^{-1}$, $k_{\mathrm{d}}=5.30 \mathrm{~s}^{-1}, k_{\mathrm{f}}=0.093 \mathrm{~s}^{-1}$ and $R C=0.25 \mathrm{~s}$. (b) $\mathrm{pH}$ effect on the photocurrent magnitude of the ITO/Ti(O)/PPIX-FCA electrode at various applied potentials. 


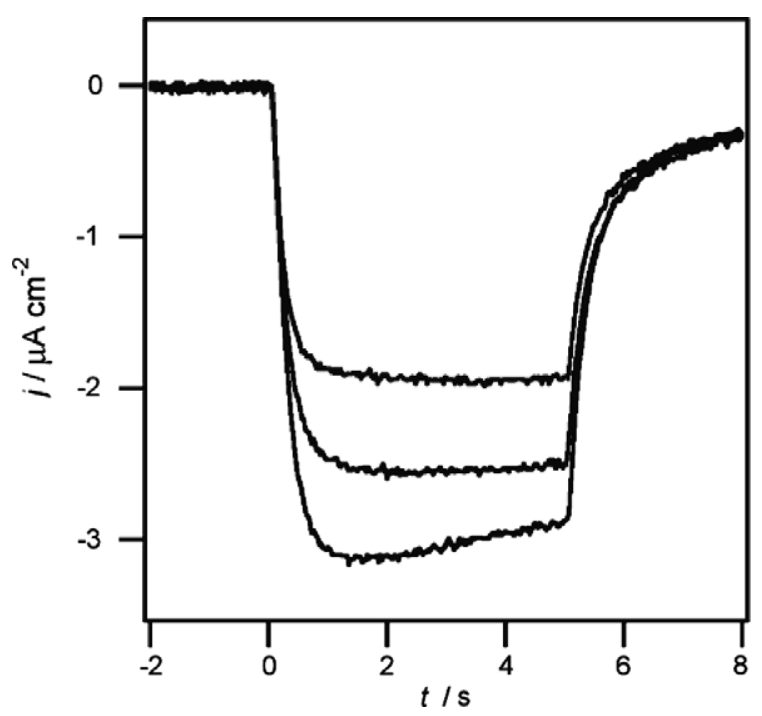

Fig. 14. The influence of solution $\mathrm{pH}(4.0,6.0$ and 9.0 from the bottom to top) on the photocurrent transient of the ITO/Ti(O)/PPIX-FCA electrode at $-0.40 \mathrm{~V}$ in the absence of oxygen in the solution.

oxygen sharply decrease in comparison with those in the presence of oxygen displayed in Fig. 13a. At pH 4.0 and 6.0 , the photocurrents decrease by $50 \%$. The decrease in the photocurrent magnitude is definitely ascribed to the oxygen quenching effect. Another feature observed in Fig. 14 is that in the absence of oxygen the photocurrent magnitude is dependent on the solution $\mathrm{pH}$. This maybe the evidence of proton reduction as discussed above. However, further investigation on $\mathrm{pH}$ effects on the photocurrent responses at much lower and higher $\mathrm{pH}$ found the instability of the $\mathrm{Ti}(\mathrm{O})$ gel and/or dye and redox mediator release [44].

\section{Conclusions}

Protoporphyrin IX (PPIX) sensitized titanium oxide $(\mathrm{Ti}(\mathrm{O}))$ xerogel films were prepared on indium tin oxide (ITO) electrodes using a sol-gel process. In this methodology, the PPIX and/or the electron donor, ferrocene carboxylic acid (FCA), were first complexed to titanium(IV) $n$-butoxide $\left(\mathrm{Ti}(\mathrm{OBu})_{4}\right)$ precursors. The resulting mixture was then subjected to hydrolysis to obtain a Ti-oxo-based macromolecular network doped with PPIX and/or FCA. The Ti(O) films on ITO electrodes were characterized by UV-Vis absorption, X-ray photoelectron spectroscopy, scanning electron microscopy and electrochemical techniques. Cyclic voltammetric measurement reveals that the oxidation/reduction of FCA inside the $\operatorname{Ti}(\mathrm{O})$ film is governed by a linear diffusion process of ion ingress/egress and/or electron hopping through the film, due to the insulating or poorly conducting nature of the gel structure.

Functionalized by PPIX, the films are photoactive and light illumination can induce the electron transfer processes. Photocurrent responses originating from the photoreduction/photooxidation of the PPIX species were investigated in an aqueous solution. Except that at very positive potentials where an anodic photocurrent was observed, a cathodic photocurrent was observed in most cases. Furthermore, incorporation of FCA inside the film and dissolved oxygen in the solution can both enhance the photocurrent responses, due to their electron donating/shuttling and accepting abilities, respectively. The dynamics of all these photoinduced processes were evaluated on the basis of a detailed kinetic model. The rate constants of electron transfer between the PPIX species and the ITO electrode, as well as that of the quenching of PPIX excited state by FCA inside the film, were found to depend on the applied potential.

Finally, clear evidence of oxygen reduction has been obtained in this work. The investigations on the $\mathrm{pH}$ effect, the lower the $\mathrm{pH}$ the larger the photocurrent, reveal that the proton reduction may also occur besides the oxygen reduction. These two processes and the possible lithium and proton insertions, as well as the diverse possibilities of functionalization, render the $\mathrm{Ti}(\mathrm{O})$ gel structure to have potential applications in devices and sensors.

\section{Acknowledgments}

This work was supported by the Swiss National Science Foundation (200020-105486) and Ecole Polytechnique Fédérale de Lausanne (EPFL). We are grateful to Fabienne Bobard and Philippe Buffat (Centre Interdisciplinaire de Miscroscopie, EPFL) for SEM measurements, Nicolas Xanthopoulos (Laboratoire de métallurgie chimique, EPFL) for XPS measurements and Pascal Comte (Laboratoire de photonique et interfaces, EPFL) for fruitful discussion and collaboration. The technical assistance by Valérie Devaud is also acknowledged.

\section{Appendix A. Mathematical development for non-mediated photocurrent responses}

The photocurrent response of the ITO/Ti(O)/PPIX electrode, named non-mediated photocurrent, involves contributions from all the steps featuring the electron transfer across the ITO/film interface, i.e., steps 5, 6, 9 and 10. Consequently, it follows:

$j_{\mathrm{ph}}=F\left\{\left(k_{\mathrm{ox}}-k_{\mathrm{red}}\right) \Gamma_{\mathrm{S}^{*}}-k_{\mathrm{red}} \Gamma_{\mathrm{S}^{+}}+k_{\mathrm{ox}} \Gamma_{\mathrm{S}^{-}}\right\}$

where $\Gamma_{i}\left(i=\mathrm{S}^{*}, \mathrm{~S}^{+}\right.$and $\left.\mathrm{S}^{-}\right)$denotes the surface concentration of PPIX in its excited, oxidized and reduced forms, respectively. The differential equations for the concentration terms in Eq. (A.1) can be written as follows:

$$
\begin{aligned}
& \frac{\mathrm{d} \Gamma_{\mathrm{S}^{*}}}{\mathrm{~d} t}=\sigma_{\mathrm{s}} I_{0} \Gamma_{\mathrm{S}}^{0}-\left(k_{\mathrm{r}}+k_{\mathrm{ox}}+k_{\mathrm{red}}+k_{\mathrm{a}}+k_{\mathrm{d}}\right) \Gamma_{\mathrm{S}^{*}} \\
& \frac{\mathrm{d} \Gamma_{\mathrm{S}^{+}}}{\mathrm{d} t}=\left(k_{\mathrm{ox}}+k_{\mathrm{a}}\right) \Gamma_{\mathrm{S}^{*}}-\left(k_{\mathrm{red}}+k_{\mathrm{d}}\right) \Gamma_{\mathrm{S}^{+}} \\
& \frac{\mathrm{d} \Gamma_{\mathrm{S}^{-}}}{\mathrm{d} t}=\left(k_{\mathrm{red}}+k_{\mathrm{d}}\right) \Gamma_{\mathrm{S}^{*}}-\left(k_{\mathrm{ox}}+k_{\mathrm{a}}\right) \Gamma_{\mathrm{S}^{-}}
\end{aligned}
$$

where $I_{0}$ is the incident photon flux. $\sigma_{\mathrm{s}}$ and $\Gamma_{\mathrm{S}}^{0}$ denote the light absorption cross-section and the surface concentration of 
the entrapped PPIX in the ground state, respectively. Using a steady-state approximation, solving Eq. (A.2) yields:

$\Gamma_{\mathrm{S}^{*}}=\frac{A I_{0}}{N_{\mathrm{A}}\left(k_{\mathrm{r}}+k_{\mathrm{ox}}+k_{\text {red }}+k_{\mathrm{a}}+k_{\mathrm{d}}\right)}$

where $A$ and $N_{\mathrm{A}}$ are the light absorption of entrapped PPIX and the Avogadro's constant, respectively. And solving Eqs. (A.3) and (A.4) gives:

$\Gamma_{\mathrm{S}^{+}}=\frac{\Gamma_{\mathrm{S}^{*}}\left(k_{\mathrm{ox}}+k_{\mathrm{a}}\right) \mathrm{e}^{-\left(k_{\mathrm{red}}+k_{\mathrm{d}}\right) t}}{k_{\mathrm{red}}+k_{\mathrm{d}}}$

$\Gamma_{\mathrm{S}^{-}}=\frac{\Gamma_{\mathrm{S}^{*}}\left(k_{\mathrm{red}}+k_{\mathrm{d}}\right) \mathrm{e}^{-\left(k_{\mathrm{ox}}+k_{\mathrm{a}}\right) t}}{k_{\mathrm{ox}}+k_{\mathrm{a}}}$

Introducing Eqs. (A.5), (A.6) and (A.7) into Eq. (A.1) produces:

$j_{\mathrm{ph}}=g\left\{a_{1}+a_{2} \mathrm{e}^{-\left(k_{\mathrm{ox}}+k_{\mathrm{a}}\right) t}+a_{3} \mathrm{e}^{-\left(k_{\mathrm{red}}+k_{\mathrm{d}}\right) t}\right\}$

where

$g=F \frac{A I_{0}}{N_{\mathrm{A}}}$

$a_{1}=\frac{k_{\mathrm{ox}}-k_{\mathrm{red}}}{k_{\mathrm{r}}+k_{\mathrm{ox}}+k_{\mathrm{red}}+k_{\mathrm{a}}+k_{\mathrm{d}}}-a_{2}+a_{3}$

$a_{2}=-\frac{k_{\text {ox }}\left(k_{\text {red }}+k_{\mathrm{d}}\right)}{\left(k_{\text {ox }}+k_{\mathrm{a}}\right)\left(k_{\mathrm{r}}+k_{\mathrm{ox}}+k_{\text {red }}+k_{\mathrm{a}}+k_{\mathrm{d}}\right)}$

$a_{3}=\frac{k_{\text {red }}\left(k_{\text {ox }}+k_{\mathrm{a}}\right)}{\left(k_{\text {red }}+k_{\mathrm{d}}\right)\left(k_{\mathrm{r}}+k_{\mathrm{ox}}+k_{\text {red }}+k_{\mathrm{a}}+k_{\mathrm{d}}\right)}$

It should also be considered that the photocurrent responses upon illumination are attenuated by the $R C$ component at the interface. Introducing this parameter into Eq. (A.8) yields the photocurrent in the Laplace plane: $\overline{j_{\mathrm{ph}}}=\left(\frac{g}{1+R C s}\right)\left\{\frac{a_{1}}{s}+\frac{a_{2}}{s+k_{\mathrm{ox}}+k_{\mathrm{a}}}+\frac{a_{3}}{s+k_{\mathrm{red}}+k_{\mathrm{d}}}\right\}$

The inverse Laplace transform of Eq. (A.13) yields Eq. (14) describing the photocurrent responses of the ITO/Ti(O)/ PPIX electrode with

$$
\begin{aligned}
\alpha= & a_{1} \\
\beta= & \frac{a_{2}}{1-\left(k_{\mathrm{ox}}+k_{\mathrm{a}}\right) R C} \\
\gamma= & \frac{a_{3}}{1-\left(k_{\mathrm{red}}+k_{\mathrm{d}}\right) R C} \\
\chi= & \frac{1}{\left[1-\left(k_{\mathrm{ox}}+k_{\mathrm{a}}\right) R C\right]\left[1-\left(k_{\mathrm{red}}+k_{\mathrm{d}}\right) R C\right]} \\
& \times\left\{-\left(a_{1}+a_{2}+a_{3}\right)+R C\left[\left(a_{1}+a_{2}\right)\left(k_{\mathrm{red}}+k_{\mathrm{d}}\right)\right.\right. \\
& \left.\left.+\left(a_{1}+a_{3}\right)\left(k_{\mathrm{ox}}+k_{\mathrm{a}}\right)\right]-R C^{2} a_{1}\left(k_{\mathrm{ox}}+k_{\mathrm{a}}\right)\left(k_{\mathrm{red}}+k_{\mathrm{d}}\right)\right\} .
\end{aligned}
$$

\section{Appendix B. Mathematical development for mediated photocurrent responses}

In the presence of FCA inside the $\mathrm{Ti}(\mathrm{O})$ structure, the photocurrent called mediated photocurrent here can be described as:

$$
j_{\mathrm{ph}}=F\left\{\left(k_{\mathrm{ox}}-k_{\mathrm{red}}\right) \Gamma_{\mathrm{S}^{*}}-k_{\mathrm{red}} \Gamma_{\mathrm{S}^{+}}+k_{\mathrm{ox}} \Gamma_{\mathrm{S}^{-}}\right.
$$

$$
\left.-k_{\mathrm{f}} \Gamma_{\mathrm{D}^{+}}\right\}
$$

The differential equation for $\mathrm{FCA}^{+}$can be written as:

$\frac{\mathrm{d} \Gamma_{\mathrm{D}^{+}}}{\mathrm{d} t}=k_{\mathrm{d}}\left(\Gamma_{\mathrm{S}^{*}}+\Gamma_{\mathrm{S}^{+}}\right)-k_{\mathrm{f}} \Gamma_{\mathrm{D}^{+}}$

Combining Eqs. (A.5), (A.6) and (B.2), $\Gamma_{\mathrm{D}^{+}}$is obtained as:

$$
\begin{aligned}
\Gamma_{\mathrm{D}^{+}}= & \Gamma_{\mathrm{S}^{*}}\left\{\frac{k_{\mathrm{d}}\left(k_{\mathrm{ox}}+k_{\text {red }}+k_{\mathrm{a}}+k_{\mathrm{d}}\right)}{k_{\mathrm{f}}\left(k_{\text {red }}+k_{\mathrm{d}}\right)}\right. \\
& +\frac{k_{\mathrm{d}}\left(k_{\text {ox }}+k_{\mathrm{a}}\right) \mathrm{e}^{-\left(k_{\text {red }}+k_{\mathrm{d}}\right) t}}{\left(k_{\text {red }}+k_{\mathrm{d}}\right)\left(k_{\text {red }}+k_{\mathrm{d}}-k_{\mathrm{f}}\right)} \\
& \left.+\frac{k_{\mathrm{d}}\left(k_{\mathrm{ox}}+k_{\text {red }}+k_{\mathrm{a}}+k_{\mathrm{d}}-k_{\mathrm{f}}\right) \mathrm{e}^{-k_{\mathrm{f}} t}}{k_{\mathrm{f}}\left(k_{\text {red }}+k_{\mathrm{d}}-k_{\mathrm{f}}\right)}\right\}
\end{aligned}
$$

Introducing Eqs. (A.5), (A.6), (A.7) and (B.3) into Eq. (B.1) yields:

$j_{\mathrm{ph}}=g\left\{c_{1}+c_{2} \mathrm{e}^{-\left(k_{\mathrm{ox}}+k_{\mathrm{a}}\right) t}+c_{3} \mathrm{e}^{-\left(k_{\mathrm{red}}+k_{\mathrm{d}}\right) t}+c_{4} \mathrm{e}^{-k_{\mathrm{f}} t}\right\}$

where

$c_{1}=-\frac{k_{\mathrm{a}}\left(k_{\mathrm{ox}}+k_{\mathrm{red}}+k_{\mathrm{a}}+k_{\mathrm{d}}\right)}{\left(k_{\mathrm{ox}}+k_{\mathrm{a}}\right)\left(k_{\mathrm{r}}+k_{\mathrm{ox}}+k_{\mathrm{red}}+k_{\mathrm{a}}+k_{\mathrm{d}}\right)}$

$c_{2}=-\frac{k_{\text {ox }}\left(k_{\text {red }}+k_{\mathrm{d}}\right)}{\left(k_{\mathrm{ox}}+k_{\mathrm{a}}\right)\left(k_{\mathrm{r}}+k_{\mathrm{ox}}+k_{\mathrm{red}}+k_{\mathrm{a}}+k_{\mathrm{d}}\right)}$

$c_{3}=\frac{\left(k_{\mathrm{ox}}+k_{\mathrm{a}}\right)\left(k_{\mathrm{red}}-k_{\mathrm{f}}\right)}{\left(k_{\mathrm{red}}+k_{\mathrm{d}}-k_{\mathrm{f}}\right)\left(k_{\mathrm{r}}+k_{\mathrm{ox}}+k_{\mathrm{red}}+k_{\mathrm{a}}+k_{\mathrm{d}}\right)}$

$c_{4}=-\frac{k_{\mathrm{d}}\left(k_{\mathrm{ox}}+k_{\mathrm{red}}+k_{\mathrm{a}}+k_{\mathrm{d}}-k_{\mathrm{f}}\right)}{\left(k_{\mathrm{red}}+k_{\mathrm{d}}-k_{\mathrm{f}}\right)\left(k_{\mathrm{r}}+k_{\mathrm{ox}}+k_{\mathrm{red}}+k_{\mathrm{a}}+k_{\mathrm{d}}\right)}$

Further taking the effect of $R C$ component into account, the photocurrent in the Laplace plane is expressed as:

$\overline{j_{\mathrm{ph}}}=\left(\frac{g}{1+R C s}\right)\left\{\frac{c_{1}}{s}+\frac{c_{2}}{s+k_{\mathrm{ox}}+k_{\mathrm{a}}}+\frac{c_{3}}{s+k_{\mathrm{red}}+k_{\mathrm{d}}}+\frac{c_{4}}{s+k_{\mathrm{f}}}\right\}$

Inverse Laplace transformation of Eq. (B.9), one obtains Eq. (15) with

$$
\begin{aligned}
\delta & =c_{1} \\
\phi & =\frac{c_{2}}{1-\left(k_{\mathrm{ox}}+k_{\mathrm{a}}\right) R C} \\
\rho & =\frac{c_{3}}{1-\left(k_{\mathrm{red}}+k_{\mathrm{d}}\right) R C} \\
\xi & =\frac{c_{4}}{1-k_{\mathrm{f}} R C} \\
\zeta= & -\frac{1}{\left[1-\left(k_{\mathrm{ox}}+k_{\mathrm{a}}\right) R C\right]\left[1-\left(k_{\mathrm{red}}+k_{\mathrm{d}}\right) R C\right]\left(1-k_{\mathrm{f}} R C\right)} \\
& \times\left\{\left(c_{1}+c_{2}+c_{3}+c_{4}\right)-R C\left[\left(k_{\mathrm{ox}}+k_{\mathrm{a}}\right)\left(c_{1}+c_{3}+c_{4}\right)\right.\right. \\
& \left.+\left(k_{\mathrm{red}}+k_{\mathrm{d}}\right)\left(c_{1}+c_{2}+c_{4}\right)+k_{\mathrm{f}}\left(c_{1}+c_{2}+c_{3}\right)\right] \\
& +R C^{2}\left[k_{\mathrm{f}}\left(k_{\mathrm{ox}}+k_{\mathrm{a}}\right)\left(c_{1}+c_{3}\right)+k_{\mathrm{f}}\left(k_{\mathrm{red}}+k_{\mathrm{d}}\right)\left(c_{1}+c_{2}\right)\right. \\
& \left.\left.+\left(k_{\mathrm{ox}}+k_{\mathrm{a}}\right)\left(k_{\mathrm{red}}+k_{\mathrm{d}}\right)\left(c_{1}+c_{4}\right)\right]-R C^{3} c_{1} k_{\mathrm{f}}\left(k_{\mathrm{ox}}+k_{\mathrm{a}}\right)\left(k_{\mathrm{red}}+k_{\mathrm{d}}\right)\right\}
\end{aligned}
$$




\section{Appendix C. Supplementary material}

Supplementary data associated with this article can be found, in the online version, at doi:10.1016/j.ica.2007. 06.036 .

\section{References}

[1] B. O'Regan, M. Graetzel, Nature 353 (1991) 737.

[2] A. Hagfeldt, M. Graetzel, Chem. Rev. 95 (1995) 49.

[3] M. Fujihira, K. Aoki, S. Inoue, H. Takemura, H. Muraki, S. Aoyagui, Thin Solid Films 132 (1985) 221.

[4] M. Fujihira, K. Nishiyama, H. Yamada, Thin Solid Films 132 (1985) 77.

[5] Y. Nishikata, A. Morikawa, M. Kakimoto, Y. Imai, Y. Hirata, K. Nishiyama, M. Fujihira, J. Chem. Soc., Chem. Commun. (1989) 1772.

[6] R. Argazzi, C.A. Bignozzi, T.A. Heimer, F.N. Castellano, G.J. Meyer, J. Phys. Chem. B 101 (1997) 2591.

[7] R. Argazzi, C.A. Bignozzi, T.A. Heimer, G.J. Meyer, Inorg. Chem. 36 (1997) 2.

[8] H. Imahori, H. Norieda, S. Ozawa, K. Ushida, H. Yamada, T. Azuma, K. Tamaki, Y. Sakata, Langmuir 14 (1998) 5335.

[9] G. Steinberg-Yfrach, J.-L. Rigaud, E.N. Durantini, A.L. Moore, D. Gust, T.A. Moore, Nature 392 (1998) 479.

[10] P. Bonhote, J.-E. Moser, R. Humphry-Baker, N. Vlachopoulos, S.M. Zakeeruddin, L. Walder, M. Graetzel, J. Am. Chem. Soc. 121 (1999) 1324.

[11] N. Armaroli, F. Barigelletti, P. Ceroni, J.-F. Eckert, J.-F. Nicoud, J.F. Nierengarten, Chem. Commun. (2000) 599.

[12] J.F. Eckert, J.-F. Nicoud, J.-F. Nierengarten, S.-G. Liu, L. Echegoyen, F. Barigelletti, N. Armaroli, L. Ouali, V. Krasnikov, G. Hadziioannou, J. Am. Chem. Soc. 122 (2000) 7467.

[13] D. Hirayama, T. Yamashiro, K. Takimiya, Y. Aso, T. Otsubo, H. Norieda, H. Imahori, Y. Sakata, Chem. Lett. (2000) 570.

[14] H. Imahori, H. Yamada, Y. Nishimura, I. Yamazaki, Y. Sakata, J. Phys. Chem. B 104 (2000) 2099.

[15] E. Peeters, P.A. van Hal, J. Knol, C.J. Brabec, N.S. Sariciftci, J.C. Hummelen, R.A.J. Janssen, J. Phys. Chem. B 104 (2000) 10174.

[16] P.A. van Hal, E.H.A. Beckers, E. Peeters, J.J. Apperloo, R.A.J. Janssen, Chem. Phys. Lett. 328 (2000) 403.

[17] E.H. Yonemoto, Y.I. Kim, R.H. Schmehl, J.O. Wallin, B.A. Shoulders, B.R. Richardson, J.F. Haw, T.E. Mallouk, J. Am. Chem. Soc. 116 (1994) 10557.

[18] T. Akiyama, H. Imahori, Y. Sakata, Chem. Lett. (1994) 1447.

[19] H. Imahori, Y. Mori, Y. Matano, J. Photochem. Photobiol. C 4 (2003) 51.
[20] H. Imahori, S. Fukuzumi, Adv. Func. Mater. 14 (2004) 525.

[21] T. Umeyama, H. Imahori, Photosynth. Res. 87 (2006) 63.

[22] M. Morisue, N. Haruta, D. Kalita, Y. Kobuke, Chem. A Eur. J. 12 (2006) 8123

[23] T. Akiyama, K.-i. Matsuoka, T. Arakawa, K. Kakutani, A. Miyazaki, S. Yamada, Jap. J. Appl. Phys. 45 (2006) 3758.

[24] S. Nitahara, N. Terasaki, T. Akiyama, S. Yamada, Thin Solid Films 438-439 (2003) 230.

[25] T. Akiyama, A. Miyazaki, M. Sutoh, I. Ichinose, T. Kunitake, S. Yamada, Colloids Surf. A 169 (2000) 137.

[26] J. Blanchard, S. Barboux-Doeuef, J. Maquet, C. Sanchez, New J. Chem. 19 (1995) 929.

[27] U. Gbureck, J. Probst, R. Thull, J. Sol-Gel Sci. Tech. 27 (2003) 157.

[28] H. Cattey, P. Audebert, C. Sanchez, P. Hapiot, J. Phys. Chem. B 102 (1998) 1193

[29] J. Livage, C. Sanchez, J. Non-Cryst. Solids 145 (1992) 11.

[30] N. Daude, C. Gout, C. Jouanin, Phys. Rev. B 15 (1977) 3229.

[31] K. Kalyananasundaram, Photochemistry of Polypyridine and Porphyrin Complexes, Academic Press Ltd, London \& San Diego, 1992.

[32] G. Hungerford, M.R. Pereira, J.A. Ferreira, T.M.R. Viseu, A.F. Coelho, M. Isabel, C. Ferreira, K. Suhling, J. Fluorosc. 12 (2002) 397.

[33] T.M.R. Viseu, G. Hungerford, M.I.C. Ferreira, J. Phys. Chem. B 106 (2002) 1853

[34] M.R. Pereira, J.A. Ferreira, G. Hungerford, J. Photochem. Photobiol. A 172 (2005) 7.

[35] H. Cattey, P. Audebert, C. Sanchez, P. Hapiot, J. Mater. Chem. 7 (1997) 1461

[36] H. Cattey, P. Audebert, C. Sanchez, New J. Chem. 20 (1996) 1023.

[37] R. Campostrini, G. Carturan, L. Palmisano, M. Schiavello, A. Sclafani, Mater. Chem. Phys. 38 (1994) 277.

[38] D. Pletcher, S. Sotiropoulos, J. Chem. Soc., Faraday Trans. 91 (1995) 457.

[39] M. Hojeij, N. Eugster, B. Su, H.H. Girault, Langmuir 22 (2006) 10652.

[40] K.M. Kadish, K.M. Smith, R. Guilard (Eds.), The Porphyrin Handbook, Database of Redox Potentials and Binding Constants, vol. 9, Academic Press Ltd., London \& San Diego, 2000.

[41] A.I. Kuznetsov, O. Kameneva, A. Alexandrov, N. Bityurin, P. Marteau, K. Chhor, C. Sanchez, A. Kanaev, Phys. Rev. E 71 (2005) $021403 / 1$

[42] I. Okura, Photosensitization of Porphyrins and Phthalocyanines, Gordon and Breach Science Publishers, Tokyo, 2000.

[43] J.H. Zagal, M.A. Paez, J.F. Silva, in: J.H. Zagal, F. Bedioui, J.P. Dodelet (Eds.), N4-macrocyclic Metal Complexes, Springer, New York, 2006, p. 41.

[44] S.W. Lee, D.H. Yang, T. Kunitake, Sensors Actuators B 104 (2005) 35. 